\title{
Low-Scaling GW with Benchmark Accuracy and Application to Phosphorene Nanosheets
}

\author{
Jan Wilhelm,* Patrick Seewald, and Dorothea Golze
}

Cite This: J. Chem. Theory Comput. 2021, 17, 1662-1677

Read Online

ABSTRACT: $G W$ is an accurate method for computing electron addition and removal energies of molecules and solids. In a conventional $G W$ implementation, however, its computational cost is $O\left(N^{4}\right)$ in the system size $N$, which prohibits its application to many systems of interest. We present a low-scaling $G W$ algorithm with notably improved accuracy compared to our previous algorithm [J. Phys. Chem. Lett. 2018, 9, 306-312]. This is demonstrated for frontier orbitals using the GW100 benchmark set, for which our algorithm yields a mean absolute deviation of only $6 \mathrm{meV}$ with respect to canonical implementations. We show that also excitations of deep valence, semicore, and unbound states CP2K: Low-scaling GW accuracy on GW100 match conventional schemes within $0.1 \mathrm{eV}$. The high accuracy is

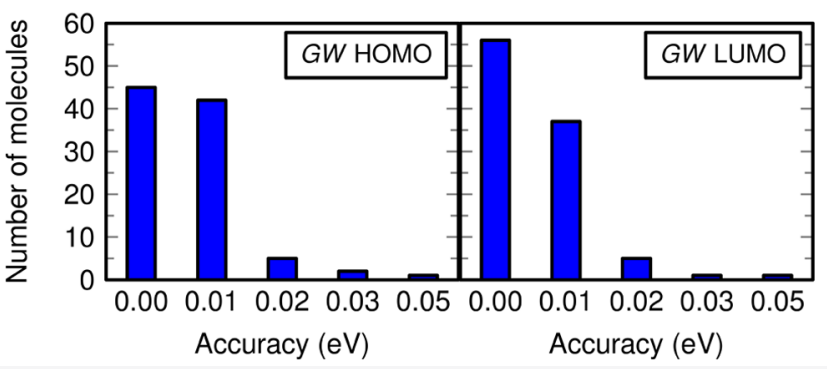
achieved by using minimax grids with 30 grid points and the resolution of the identity with the truncated Coulomb metric. We apply the low-scaling $G W$ algorithm with improved accuracy to phosphorene nanosheets of increasing size. We find that their fundamental gap is strongly size-dependent varying from $4.0 \mathrm{eV}(1.8 \mathrm{~nm} \times 1.3 \mathrm{~nm}, 88$ atoms $)$ to $2.4 \mathrm{eV}(6.9 \mathrm{~nm} \times 4.8 \mathrm{~nm}, 990$ atoms $)$ at the evGW $W_{0} @ P B E$ level.

\section{INTRODUCTION}

The GW approximation ${ }^{1}$ to many-body perturbation theory has become the method of choice for the calculation of photoemission spectra of materials and more recently also of molecules. ${ }^{2,3}$ The extension of $G W$ to the Bethe-Salpeter equation ${ }^{4}$ has been extensively applied for the accurate computation of absorption spectra in materials science 5 and chemistry $^{6,7}$ and lately also to ground- and excited-state geometry optimizations. ${ }^{8,9}$ Recent $G W$ trends include the application to deep core excitations, ${ }^{10-14}$ comprehensive benchmarking, ${ }^{15-22}$ and the development of computationally efficient schemes for large-scale calculations of systems with $\geq 1000$ atoms. ${ }^{19,23,24}$ This work contributes to the last two points with a focus on avoiding the loss of numerical accuracy with respect to canonical GW implementations.

The application of conventional $G W$ schemes is restricted to systems with a few hundred atoms, ${ }^{25,26}$ due to the $O\left(N^{4}\right)$ scaling with respect to system size $N$ and the large overall computational cost (prefactor). Recent developments to make larger system sizes computationally tractable cover the range from massively parallel implementations over physically motivated approximations to novel numerical methods. Efficient parallelization schemes were developed for execution on more than 10000 CPU cores, ${ }^{19,23,27-29}$ and first algorithms have been already proposed for the new generation of heavily GPU-based (pre)exascale supercomputers. ${ }^{30}$ An example for more physically motivated developments are $G W$ embedding schemes, where a small part of the system is calculated at the $G W$ level and the surrounding medium is treated at a lower level of theory. ${ }^{31-33}$ Numerical developments have proceeded in several directions, reducing either the computational prefactor or the scaling with respect to system size.

The prefactor has been reduced by avoiding the summation over unoccupied states by solving the Sternheimer equation. ${ }^{34-39}$ A different strategy to reduce the overall computational cost is low-rank approximations of the polarizability, which map the latter onto a smaller basis. ${ }^{23,38,40,41}$ Others addressed the frequency integration ${ }^{42-44}$ or explored real-space density fitting schemes. ${ }^{22}$ The size of the matrices can be also reduced by choosing an optimal basis set for the respective problem. Localized basis sets are generally smaller than traditional plane-wave basis sets and particularly suited for molecular systems. The implementation of $G W$ in quantum chemistry codes, which typically use localized basis sets, is a rather recent development of the past decade. ${ }^{19,21,25,45-49}$ The efficient inclusion of periodic boundary conditions into algorithms with localized basis sets is still the subject of ongoing work. ${ }^{14,50-53}$

Received: December 11, 2020

Published: February 23, 2021 
Scaling reduction is a particularly promising approach when aiming at applications to nanostructured systems, which require very large system sizes with 1000 atoms and more. Different approaches have been explored for the reduction of the scaling with respect to the system size. A linear scaling algorithm was devised within the framework of stochastic $G W .^{24}$ While the stochastic schemes have been successfully applied to silicon, ${ }^{24}$ the application to molecules seems to be more challenging. ${ }^{54}$ Several cubic-scaling algorithms were developed, ${ }^{19,21,55-58}$ which are based on or at least inspired by the space-time method proposed by Rojas, Godby, and Needs in $19955^{59}$ Variants of the space-time method have been implemented in a plane-wave/projector-augmented-wave (PAW) $G W \operatorname{code}^{56}$ and also with localized basis sets using Gaussian ${ }^{19,57}$ and Slater-type orbitals. $^{21}$

In our recent work, ${ }^{19}$ we devised a low-scaling $G W$ algorithm in a Gaussian basis with formal $O\left(N^{3}\right)$ complexity, which has been optimized for massively parallel execution. Sparse linear algebra was exploited by using the resolution-of-the-identity (RI) approach with an overlap metric to refactor the four-center electron repulsion integrals. We showed that our algorithm effectively scales $O\left(N^{2}\right)$, and we applied it to quasi-onedimensional systems (graphene nanoribbons) with more than 1700 atoms and 5700 electrons. An important property of lowscaling algorithms is the crossover point. The latter refers to the system size, where the low-scaling algorithm, which has usually a larger computational prefactor, becomes computationally more efficient than the canonical scheme. We demonstrated that the crossover point is already at around 150 atoms. ${ }^{19}$

Another challenge for low-scaling $G W$ algorithms is reaching high numerical accuracy. ${ }^{21,54}$ The GW100 benchmark ${ }^{15}$ has set the accuracy standards for molecules. Using identical basis sets, it was demonstrated that it is possible to match $G W$ excitations of the highest occupied molecular orbital (HOMO) and lowest unoccupied molecular orbital (LUMO) within $<10 \mathrm{meV}^{15}$ between two $G W$ implementations ${ }^{46,47}$ based on numerically very different techniques. For our previous low-scaling algorithm, ${ }^{19}$ we found that the $G W 100$ mean absolute deviation (MAD) with respect to the canonical reference implementation in FHI-aims ${ }^{46}$ is $35 \mathrm{meV}$ for ionization potentials and $27 \mathrm{meV}$ for electron affinities. In addition, a couple of outliers with deviations in the range of $200 \mathrm{meV}$ were observed; see ref 19 (Supporting Information) and ref 2 for a comparison of the accuracy of different implementations.

The goal of this work is to increase the accuracy of the lowscaling $G W$ algorithm toward benchmark accuracy, i.e., MADs of less than $10 \mathrm{meV}$ for the GW100 test, while retaining high computational efficiency. Furthermore, we aim to increase the reliability of our algorithm by reducing the number of outliers. High accuracy is achieved by a twofold approach. The first is an increase and dedicated optimization of the minimax time and frequency grids, which can be directly transferred to other implementations of the space-time method. Second, we replace the overlap RI metric by the truncated Coulomb metric (RI-tC). In this work, the RI-tC approach is explored in the context of $G W$ for the first time.

The remainder of this article is organized as follows: In Section 2, the $G W$ space-time method ${ }^{9,60}$ is introduced in a real-space grid formulation for nonperiodic systems. The RI-tC approach is discussed in Section 3. Combining both the GW space-time method and the RI-tC within a Gaussian basis, we arrive at our low-scaling GW algorithm (Section 4). Implementation details and computational details are given in
Sections 5 and 6, respectively. Convergence tests of the minimax grid and the RI-tC are reported in Section 7, including benchmark studies for the GW100 test set. We demonstrate that our low-scaling algorithm is accurate not only for frontier orbitals but also for semicore and unbound states by comparing to highly accurate contour-deformation results from the FHIaims code ${ }^{11}$ in Section 8. We apply our new low-scaling scheme to compute fundamental gaps of phosphorene nanosheets, which show potential as novel two-dimensional semiconductors, in Section 9. Finally, we discuss the computational efficiency of our implementation in Section 10 and draw conclusions in Section 11.

\section{GW SPACE-TIME METHOD IN A REAL-SPACE FORMULATION}

The GW space-time method was proposed by Rojas, Godby, and Needs in 1995, ${ }^{59}$ enabling the computation of $G W$ quasiparticle (QP) energies at $O\left(N^{3}\right)$ complexity. The approach by Rojas et al. targets the application to solids employing a real-space grid in combination with a plane-wave basis. Fast Fourier transforms are used to change the representation from the real-space grid to plane waves, introducing a large computational prefactor. To keep the computational cost tractable, the original space-time approach is typically used together with soft pseudopotentials. ${ }^{60}$ This implies that deep valence or semicore states are not included in the calculation of the density response functions, making the application to materials with, e.g., localized $d$ electrons difficult.

The GW space-time method was adapted to the PAW methodology by Liu et al. in 2016, ${ }^{56}$ enabling the inclusion of more localized states in the density response function. The PAW implementation in VASP allows the efficient treatment of molecules ${ }^{16}$ and large supercells ${ }^{56}$ with high accuracy. However, the large computational prefactor due to the fast Fourier transforms between real and reciprocal space remains, similarly as in the original method. ${ }^{59}$

Fast Fourier transforms can be circumvented by replacing the real-space grid and the plane-waves basis by a localized basis, which was first explored in our work from $2018^{19}$ and very recently also by Förster and Visscher. ${ }^{21}$ In our work from 2018, we used a Gaussian basis in combination with a local metric (overlap) for the RI refactorization of the four-center Coulomb integrals. The low-scaling $G W$ algorithm developed by Förster and Visscher ${ }^{21}$ employs Slater-type functions. Unlike in our approach, sparsity is introduced by a local RI scheme (pairatomic density fitting) instead of a local metric. We elaborate on the difference, advantages, and disadvantages in Section 3.3.

An alternative reformulation of the space-time method was proposed by Duchemin and Blase, ${ }^{57}$ combining a real-space grid with a Gaussian basis instead of plane waves. The real-space grid is specifically optimized for the respective molecule by the separable resolution of the identity. In ref 57, the described approach was only applied to the random phase approximation (RPA), but the extension to $G W$ is straightforward.

The aforementioned space-time algorithms ${ }^{19,21,56,57,59,60}$ differ in the choice of the basis and the associated numerical techniques. However, the time and frequency treatments are identical. To introduce the basic equations, we start with a generic reformulation of the $G W$ space-time algorithm for nonperiodic systems projecting all quantities on real-space grids. Note that these generic expressions differ from the original work by Rojas et al., 59 where only some quantities are computed on real-space grids, e.g., the polarizability, and others, e.g., the 
dielectric function, in a plane-wave basis. In Sections 3 and 4, we will project these generic expressions into a Gaussian basis.

We start from a self-consistent Kohn-Sham density functional theory (KS-DFT) calculation. The total energy of a manyelectron system in KS-DFT is obtained by solving the eigenvalue problem

$$
\left(h^{0}(\mathbf{r})+v_{\mathrm{xc}}(\mathbf{r})\right) \psi_{n}(\mathbf{r})=\varepsilon_{n} \psi_{n}(\mathbf{r})
$$

$h_{0}(\mathbf{r})$ contains the external and the Hartree potential as well as the kinetic energy, while the exchange-correlation potential $v^{\mathrm{xc}}(\mathbf{r})$ accounts for electron-electron interaction beyond the Hartree interaction. In the $G W$ space-time method, we use molecular orbitals (MOs) $\psi_{n}(\mathbf{r})$ and eigenvalues $\varepsilon_{n}$ for computing the single-particle Green's function in imaginary time as

$$
G\left(\mathbf{r}, \mathbf{r}^{\prime}, i \tau\right)= \begin{cases}i \sum_{i}^{\text {occ }} \psi_{i}(\mathbf{r}) \psi_{i}\left(\mathbf{r}^{\prime}\right) \exp \left(\varepsilon_{i} \tau\right), & \tau>0 \\ -i \sum_{a}^{\text {virt }} \psi_{a}(\mathbf{r}) \psi_{a}\left(\mathbf{r}^{\prime}\right) \exp \left(\varepsilon_{a} \tau\right), & \tau<0\end{cases}
$$

The irreducible polarizability is computed as

$$
\chi^{0}\left(\mathbf{r}, \mathbf{r}^{\prime}, i \tau\right)=-i G\left(\mathbf{r}, \mathbf{r}^{\prime}, i \tau\right) G\left(\mathbf{r}, \mathbf{r}^{\prime},-i \tau\right)
$$

We proceed by a Fourier transform to imaginary time to evaluate the dielectric function and its inverse as

$$
\begin{aligned}
\epsilon\left(\mathbf{r}, \mathbf{r}^{\prime}, i \omega\right)= & \delta\left(\mathbf{r}, \mathbf{r}^{\prime}\right)-\int \mathrm{d} \mathbf{r}^{\prime \prime} v\left(\mathbf{r}, \mathbf{r}^{\prime \prime}\right) \chi^{0}\left(\mathbf{r}^{\prime \prime}, \mathbf{r}^{\prime}, i \omega\right) \\
\epsilon^{-1}\left(\mathbf{r}, \mathbf{r}^{\prime}, i \omega\right)= & \delta\left(\mathbf{r}, \mathbf{r}^{\prime}\right) \\
& +\int \mathrm{d} \mathbf{r}^{\prime \prime} v\left(\mathbf{r}, \mathbf{r}^{\prime \prime}\right) \chi^{0}\left(\mathbf{r}^{\prime \prime}, \mathbf{r}^{\prime}, i \omega\right)+\ldots
\end{aligned}
$$

with the bare Coulomb interaction $v\left(\mathbf{r}, \mathbf{r}^{\prime}\right)=1 /\left|\mathbf{r}-\mathbf{r}^{\prime}\right|$ and using $(1-x)^{-1}=1+x+x^{2}+\ldots$ for $|x|<1$ in eq 5 . The screened Coulomb interaction $W$ is then given by

$$
W\left(\mathbf{r}, \mathbf{r}^{\prime}, i \omega\right)=\int \mathrm{d} \mathbf{r}^{\prime \prime} \epsilon^{-1}\left(\mathbf{r}, \mathbf{r}^{\prime \prime}, i \omega\right) v\left(\mathbf{r}^{\prime \prime}, \mathbf{r}^{\prime}\right)
$$

Note that eqs 4-6 are not implemented in real-space in any of the discussed space-time algorithms because the computational cost of eqs 4-6 quickly grows as $N_{\text {grid }}^{3}$ with the number of realspace grid points $N_{\text {grid }}$ which prohibits the application to large systems. In the original space-time method, ${ }^{59}$ eqs 4 and 6 are formulated in plane-waves with a diagonal Coulomb operator $V_{\mathbf{G G}^{\prime}}=\delta_{\mathbf{G G}^{\prime}} / \mid \mathbf{G}^{2}$ such that the scaling of eqs 4 and 6 is reduced to $O\left(N^{2}\right)$.

We continue the algorithm by a Fourier transform of $W(i \omega)$ from eq 6 to imaginary time to evaluate the self-energy as

$$
\Sigma\left(\mathbf{r}, \mathbf{r}^{\prime}, i \tau\right)=i G\left(\mathbf{r}, \mathbf{r}^{\prime}, i \tau\right) W\left(\mathbf{r}, \mathbf{r}^{\prime}, i \tau\right)
$$

After Fourier transforming $\Sigma(i \tau)$ to imaginary frequency $i \omega$, we use analytic continuation to obtain $\Sigma(\omega)$ such that the $G_{0} W_{0}$ QP energies can be evaluated as

$$
\varepsilon_{n}^{G_{0} W_{0}}=\varepsilon_{n}+\operatorname{Re} \Sigma_{n}\left(\varepsilon_{n}^{G_{0} W_{0}}\right)-v_{n}^{\mathrm{xc}}
$$

where $v_{n}^{\mathrm{xc}}$ and $\Sigma_{n}(\varepsilon)$ are $(n, n)$-diagonal matrix elements in the MO basis $\psi_{n}$ of the respective quantities,

$$
\Sigma_{n}(\varepsilon)=\int \mathrm{d} \mathbf{r} \mathrm{d} \mathbf{r}^{\prime} \psi_{n}(\mathbf{r}) \Sigma\left(\mathbf{r}, \mathbf{r}^{\prime}, \varepsilon\right) \psi_{n}\left(\mathbf{r}^{\prime}\right)
$$

$$
v_{n}^{\mathrm{xc}}=\int \mathrm{d} \mathbf{r} \psi_{n}(\mathbf{r}) v^{\mathrm{xc}}(\mathbf{r}) \psi_{n}(\mathbf{r})
$$

In this work, we will also use eigenvalue-self-consistent $G W_{0}$ $\left(\right.$ ev $\left.G W_{0}\right)$ where $\varepsilon_{n}^{G_{0} W_{0}}$ are used to recompute $G(i \tau)$ from eq 2 . $\Sigma(i \tau)$ follows from eq 7 using $W(i \tau)$ from $G_{0} W_{0}$. The QP energy is recomputed from eq 8 . In evGW $W_{0}$, this cycle is repeated until the QP energies are converged.

The scaling of the different steps is summarized in Figure 1. In a canonical implementation, the evaluation of the polarizability

(a)

$$
\begin{array}{r}
\chi^{0}\left(\mathbf{r}, \mathbf{r}^{\prime}, i \omega\right)=\sum_{i}^{\text {occ }} \sum_{a}^{\text {virt }} \frac{2\left(\varepsilon_{i}-\varepsilon_{a}\right)}{\left(\varepsilon_{i}-\varepsilon_{a}\right)^{2}+\omega^{2}} \psi_{i}(\mathbf{r}) \psi_{a}(\mathbf{r}) \psi_{i}\left(\mathbf{r}^{\prime}\right) \psi_{a}\left(\mathbf{r}^{\prime}\right) \\
\mathcal{O}\left(N_{\text {grid }}^{2} N_{\text {occ }} N_{\text {virt }}\right)=\mathcal{O}\left(N^{4}\right)
\end{array}
$$

(b)

$$
\begin{gathered}
G\left(\mathbf{r}, \mathbf{r}^{\prime}, i \tau\right)=\left\{\begin{array}{cc}
i \sum_{i}^{\text {occ }} \psi_{i}(\mathbf{r}) \psi_{i}\left(\mathbf{r}^{\prime}\right) \exp \left(\varepsilon_{i} \tau\right), & \tau>0 \\
-i \sum_{a}^{\text {virt }} \psi_{a}(\mathbf{r}) \psi_{a}\left(\mathbf{r}^{\prime}\right) \exp \left(\varepsilon_{a} \tau\right), & \tau<0
\end{array}\right. \\
\mathcal{O}\left(N_{\text {grid }}^{2}\left(N_{\text {occ }}+N_{\text {virt }}\right)\right)=\mathcal{O}\left(N^{3}\right) \\
\chi^{0}\left(\mathbf{r}, \mathbf{r}^{\prime}, i \tau\right)=-i G\left(\mathbf{r}, \mathbf{r}^{\prime}, i \tau\right) G\left(\mathbf{r}, \mathbf{r}^{\prime},-i \tau\right) \\
\mathcal{O}\left(N_{\text {grid }}^{2}\right)=\mathcal{O}\left(N^{2}\right) \\
\chi^{0}\left(\mathbf{r}, \mathbf{r}^{\prime}, i \omega\right)=\mathrm{FT}\left[\chi^{0}\left(\mathbf{r}, \mathbf{r}^{\prime}, i \tau\right)\right]
\end{gathered}
$$

Figure 1. Computation of the irreducible polarizability (a) in an ordinary $O\left(\mathrm{~N}^{4}\right)$ implementation ${ }^{2}$ and (b) in the $G W$ space-time method. ${ }^{59}$ In most $G W$ algorithms, this step dominates the computational cost of the whole $G W$ calculation. In (a), the computational cost increases as $N^{4}$ with the system size $N$ since the following quantities each increase linearly with $N$ : the number of real-space grid points $N_{\text {grid }}$, the number of occupied molecular orbitals $N_{\text {occ }}$ and the number of virtual molecular orbitals $N_{\text {virt }}$. In (b), we repeat eqs 2 and 3. Calculating the irreducible polarizability in imaginary frequency is reduced to $O\left(N^{3}\right)$ scaling.

is the computational bottleneck and scales with $O\left(N^{4}\right)$; see Figure 1a. The space-time method decouples the summation over occupied and virtual states in the polarizability by expressing $G$ in the time instead of the frequency domain; see eq 2. This reduces the scaling to at most cubic, as shown in Figure $1 \mathrm{~b}$.

\section{RESOLUTION OF THE IDENTITY (RI) USING THE TRUNCATED COULOMB METRIC}

3.1. RI for Four-Center Coulomb Integrals. Before reformulating the $G W$ space-time method from Section 2 in a Gaussian basis, we focus on four-center Coulomb integrals (4cCIs) that are of central importance in $G W$ calculations with localized basis sets. These 4c-CIs, in Mulliken notation, are defined as

$$
(\mu \nu \mid \lambda \sigma):=\int \mathrm{d} \mathbf{r} \mathrm{d} \mathbf{r}^{\prime} \phi_{\mu}\left(\mathbf{r}^{\prime}\right) \phi_{\nu}\left(\mathbf{r}^{\prime}\right) \phi_{\lambda}(\mathbf{r}) \phi_{\sigma}(\mathbf{r}) \frac{1}{\left|\mathbf{r}-\mathbf{r}^{\prime}\right|}
$$


where $\phi_{\mu}, \phi_{\nu}, \phi_{\lambda}$, and $\phi_{\sigma}$ are atomic-orbital (AO) Gaussian basis functions. Using the $\mathrm{RI}^{61-63}$ approximation with a predefined metric

$$
m: \mathbb{R}^{3} \times \mathbb{R}^{3} \rightarrow[0, \infty)
$$

the $4 \mathrm{c}$-CIs are factorized into two- and three-center integrals ${ }^{61}$

$$
(\mu \nu \mid \lambda \sigma)_{\mathrm{RI}}=\sum_{P Q R S}(\mu \nu \mid P)_{m} M_{P Q}^{-1} V_{Q R} M_{R S}^{-1}(S \mid \lambda \sigma)_{m}
$$

$P, Q R$, and $S$ refer to indices of auxiliary RI Gaussian basis functions. $\mathbf{M}$ denotes the representation of the metric $m$ in the auxiliary RI basis $\left\{\varphi_{P}\right\}$,

$$
M_{P Q}=\int \mathrm{d} \mathbf{r} \mathrm{d} \mathbf{r}^{\prime} \varphi_{P}(\mathbf{r}) m\left(\mathbf{r}, \mathbf{r}^{\prime}\right) \varphi_{Q}\left(\mathbf{r}^{\prime}\right)
$$

The three-center integrals $(\mu \nu \mid P)_{m}$ are given by

$$
(\mu \nu \mid P)_{m} \equiv(P \mid \mu \nu)_{m}=\int \mathrm{d} \mathbf{r} \mathrm{d} \mathbf{r}^{\prime} \phi_{\mu}(\mathbf{r}) \phi_{\nu}(\mathbf{r}) m\left(\mathbf{r}, \mathbf{r}^{\prime}\right) \varphi_{P}\left(\mathbf{r}^{\prime}\right)
$$

The bare Coulomb interaction $1 /\left|\mathbf{r}-\mathbf{r}^{\prime}\right|$ from eq 11 is contained in the Coulomb matrix element $V_{Q R}$ in eq 13 which is given by

$$
V_{P Q}=\int \mathrm{d} \mathbf{r} \mathrm{d} \mathbf{r}^{\prime} \varphi_{P}(\mathbf{r}) \frac{1}{\left|\mathbf{r}-\mathbf{r}^{\prime}\right|} \varphi_{Q}\left(\mathbf{r}^{\prime}\right)
$$

We compute the two-center integrals in eqs 15 and 16 with a solid-harmonic-based analytical integration scheme ${ }^{64}$ and the three-center integrals from eq 15 with the analytical ObaraSaika recurrence scheme. ${ }^{65}$ Both schemes are applicable to general interaction potentials $g\left(\left|\mathbf{r}_{1}-\mathbf{r}_{2}\right|\right),{ }^{64,66}$ which includes the overlap, Coulomb, and truncated Coulomb potential discussed in Section 3.2. The calculation of the integrals starts in both schemes from integrals over primitive $s$-functions. The analytical expressions for the s-type integrals are given in ref 66 for the overlap and Coulomb potentials and in ref 67 for the truncated Coulomb potential. Prescreening of the two- and three-center integrals is applied dependent on the metric. For the truncated Coulomb metric, the integrals are screened based on the exponents, the distance between the Gaussian centers, and the truncation radius. In addition, computed three-center integrals, which are sufficiently close to zero, are filtered out before calculating the $G W$ quantities.

3.2. Truncated Coulomb Metric as a Convenient Choice in Low-Scaling Methods. The first key ingredient to reduce the scaling is the decoupling of the occupied and virtual MOs in the polarizability by working in the time domain. The second ingredient, when working in a localized basis set, is the choice of the RI metric. In our previous implementation of low-scaling $G W{ }^{19}$ we employed the overlap metric ${ }^{61}$

$$
m_{\mathrm{O}}\left(\mathbf{r}, \mathbf{r}^{\prime}\right)=\delta\left(\mathbf{r}-\mathbf{r}^{\prime}\right)
$$

for computing the integrals in eqs 14 and 15, where $\delta$ is the Dirac distribution. The overlap metric is local in the sense that the RI basis functions $\varphi_{P}$ do not overlap with $\mathrm{AO}$ basis function products $\phi_{\mu} \phi_{\nu}$ in eq 15 if there is enough distance between their centers. This leads to vanishing three-center overlap matrix elements $(\mu \nu \mid P)_{m}$ and increasing computational efficiency due to sparsity, as illustrated in Figure 2.

In contrast, the Coulomb metric

$$
m_{\mathrm{C}}\left(\mathbf{r}, \mathbf{r}^{\prime}\right)=\frac{1}{\left|\mathbf{r}-\mathbf{r}^{\prime}\right|}
$$

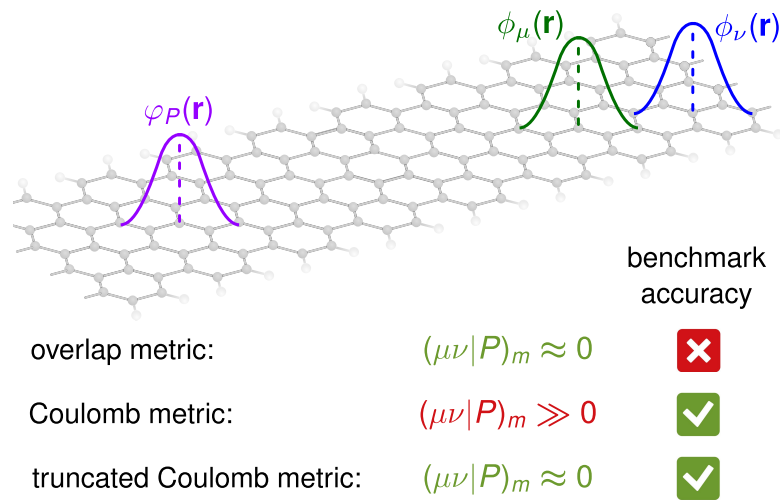

Figure 2. Sketch of three Gaussian basis functions, where the AO basis functions $\phi_{\mu}(\mathbf{r})$ and $\phi_{\nu}(\mathbf{r})$ are close together, while the RI basis function $\varphi_{P}(\mathbf{r})$ is far away from $\phi_{\mu}(\mathbf{r}), \phi_{\nu}(\mathbf{r})$. In this case, the three-center integral $(\mu \nu \mid P)_{m}$ from eq 15 vanishes in the overlap metric and in the truncated Coulomb metric, while in the Coulomb metric the threecenter integrals $(\mu \nu \mid P)_{m}$ are nonvanishing. High accuracy in electronic structure methods can only be achieved by the Coulomb metric and the truncated Coulomb metric. ${ }^{61}$

couples RI basis functions $\varphi_{P}$ and AO basis function pairs $\phi_{\mu} \phi_{\nu}$ in eq 15 over effectively infinite distances due to the slow polynomial decay of $1 /\left|\mathbf{r}-\mathbf{r}^{\prime}\right|$ as illustrated in Figure $2 .{ }^{68}$ With the Coulomb metric, no sparsity can be gained, hampering its usage in low-scaling $G W$ algorithms. In canonical $O\left(N^{4}\right)$ algorithms, each $\mathrm{AO}$ product $\phi_{\mu} \phi_{\nu}$ is transformed to the delocalized molecular orbital basis $\left\{\psi_{n}\right\}$, losing all sparsity anyway. ${ }^{25,46,50,68-76}$ In such a conventional algorithm, where sparsity cannot be exploited, the Coulomb metric is the optimal choice because the RI factorization given in eq 13 converges much quicker with respect to the RI basis set size. ${ }^{61}$ The Coulomb metric yields thus generally higher accuracy than the overlap metric.

In this work, we improve our previous low-scaling $G W$ implementation $^{19}$ by replacing the overlap metric by the truncated Coulomb metric ${ }^{68,77-79}$

$$
m_{r_{\mathrm{c}}}\left(\mathbf{r}, \mathbf{r}^{\prime}\right)= \begin{cases}\frac{1}{\left|\mathbf{r}-\mathbf{r}^{\prime}\right|} & \text { if }\left|\mathbf{r}-\mathbf{r}^{\prime}\right|<r_{\mathrm{c}} \\ 0 & \text { else }\end{cases}
$$

where the Coulomb interaction is cut after a distance $r_{\mathrm{c}}$. In the limit of a large cutoff radius $r_{\mathrm{c}}$, the truncated Coulomb metric turns into the Coulomb metric, $\lim _{r_{\mathrm{c}} \rightarrow \infty} m_{r_{\mathrm{c}}}\left(\mathbf{r}, \mathbf{r}^{\prime}\right)=1 /\left|\mathbf{r}-\mathbf{r}^{\prime}\right|$. For a small cutoff radius $r_{\mathcal{c}}$ calculations based on the truncated Coulomb metric are equivalent to calculations based on the overlap metric. ${ }^{68}$ The truncated Coulomb metric combines the attractive features of the Coulomb metric and the overlap metric: high accuracy due to the near-sighted Coulomb operator and preservation of sparsity due to the locality of $m_{r_{c}}\left(\mathbf{r}, \mathbf{r}^{\prime}\right)$.

Another approach for truncating the Coulomb operator is the use of complementary error functions as in standard rangeseparated hybrid functionals. ${ }^{80,81}$ The benefits of a local Coulomb metric have already been exploited for low-scaling scaled-opposite spin $\mathrm{MP}^{78}$ and low-scaling $\mathrm{RPA}^{68,82-83}$ reporting similar accuracy as for the respective conventionalscaling schemes.

The RI factorization in eq 13 is exact in the limit of a complete RI basis, independent of the chosen RI metric. Therefore, truncating the Coulomb operator with a finite $r_{\mathrm{c}}$ does not affect 
the accuracy of the $G W$ algorithm as long as the RI basis is sufficiently large.

We note that in plane-wave implementations, RI with different metrics is not discussed. The reason is that the Coulomb matrix, the truncated Coulomb matrix, and the overlap matrix are diagonal in the plane-wave basis. As a consequence, RI factorizations as in eq 13 are identical for the three different metrics when using plane-wave basis functions. We added a more detailed explanation in the Supporting Information (SI) to facilitate the discussion between plane-wave and localized-basis-set communities.

3.3. Global vs Local RI. The sums over the RI basis functions in the RI factorization of the $4 \mathrm{c}$ Coulomb integrals in eq 13 can either run over the whole RI basis ("global RI") or only over a subset of the RI basis ("local RI"). In their recent work, Förster and Visscher ${ }^{21}$ combined the $G W$ space-time method with the pair-atomic RI (PARI) approach. ${ }^{86}$ PARI, also known as pair-atomic density fitting (PADF) or RI-LVL, ${ }^{87}$ is a local RI approach, which employs the Coulomb metric. Locality is introduced by expanding each $\mathrm{AO}$ pair $\phi_{\mu} \phi_{\nu}$, where $\phi_{\mu}$ is centered at atom $A$ and $\phi_{\nu}$ at atom $B$, only in the subset of RI basis functions with centers at $A$ and $B$.

The scaling with PARI is the same as with global RI, if a local $\mathrm{RI}$ metric (overlap, truncated Coulomb) is employed for the latter. However, PARI reduces the computational prefactor dramatically compared to global RI since the number of threecenter integrals is substantially smaller. For example, the computational cost of a $G W$ calculation on $\approx 400$ atoms with around $8000 \mathrm{AOs}$ is $\approx 4000 \mathrm{CPU}$ hours with our low-scaling scheme using global RI with the overlap metric, ${ }^{19}$ but only $\approx 200$ CPU hours with the PARI implementation by Förster and Visscher. ${ }^{21}$ However, reaching high accuracy in low-scaling PARI-GW seems more challenging. ${ }^{21}$

The accuracy of local RI schemes can be improved by adding high-angular-momentum functions to the RI basis set and increasing its size. ${ }^{51,87}$ It has been recently shown for a local RI variant of a $G W$ implementation with conventional scaling that good accuracy can be obtained with carefully chosen RI basis sets. ${ }^{51}$ However, local RI schemes tend to be ill-conditioned problems ${ }^{88}$ due to very large RI basis sets, which might limit the attainable accuracy to some extent. It should be generally easier to reach high accuracy with $\mathrm{MADs} \leq 10 \mathrm{meV}$, which is the focus of this work, with global RI-tC rather than a local PARI-type approach.

\section{GW SPACE-TIME METHOD IN A GAUSSIAN BASIS USING RI WITH THE TRUNCATED COULOMB METRIC}

In the following, we present our low-scaling $G W$ algorithm, which is a variant of the space-time method introduced in Section 2 and rationalize where the RI factorization from eq 13 enters the algorithm.

4.1. Low-Scaling Algorithm. The MOs $\left\{\psi_{n}\right\}$ are expanded in Gaussian-type orbitals (GTOs) $\left\{\phi_{\mu}\right\}$

$$
\psi_{n}(\mathbf{r})=\sum_{\mu} C_{n \mu} \phi_{\mu}(\mathbf{r})
$$

where $C_{n \mu}$ are the $\mathrm{MO}$ coefficients. The single-particle Green's function $G(i \tau)$ given in eq 2 is then projected in the GTO basis

$$
G_{\mu \nu}(i \tau)= \begin{cases}i \sum_{n}^{\text {occ }} C_{n \mu} C_{n \nu} \exp \left(\varepsilon_{n} \tau\right), & \tau>0 \\ -i \sum_{n}^{\text {virt }} C_{n \mu} C_{n \nu} \exp \left(\varepsilon_{n} \tau\right), & \tau<0\end{cases}
$$

Next, we use $G\left(\mathbf{r}, \mathbf{r}^{\prime}, i \tau\right)=\sum_{\mu \nu} \phi_{\mu}(\mathbf{r}) G_{\mu \nu}(i \tau) \phi_{\nu}\left(\mathbf{r}^{\prime}\right)$ and eq 3, $\chi^{0}\left(\mathbf{r}, \mathbf{r}^{\prime}, i \tau\right)=-i G\left(\mathbf{r}, \mathbf{r}^{\prime}, i \tau\right) G\left(\mathbf{r}, \mathbf{r}^{\prime},-i \tau\right)$, to obtain the irreducible polarizability $\chi^{0}(i \tau)$ in the Gaussian auxiliary basis $\left\{\varphi_{P}\right\}^{27,69,71,89}$

$$
\begin{aligned}
\chi_{P Q}^{0}(i \tau):= & \left(\varphi_{P}\left|\chi^{0}\right| \varphi_{Q}\right)_{m} \\
:= & \int \mathrm{d} \mathbf{r}_{1} \mathrm{~d} \mathbf{r}_{2} \mathrm{~d} \mathbf{r}_{3} \mathrm{~d} \mathbf{r}_{4} \varphi_{P}\left(\mathbf{r}_{1}\right) m\left(\mathbf{r}_{1}, \mathbf{r}_{2}\right) \\
& \times \chi^{0}\left(\mathbf{r}_{2}, \mathbf{r}_{3}, i \tau\right) m\left(\mathbf{r}_{3}, \mathbf{r}_{4}\right) \varphi_{Q}\left(\mathbf{r}_{4}\right) \\
= & -i \sum_{\mu \sigma} \sum_{\lambda}(\lambda \sigma \mid P)_{m} G_{\mu \lambda}(i \tau) \sum_{\nu}(\mu \nu \mid Q)_{m} G_{\nu \sigma}(-i \tau)
\end{aligned}
$$

The three-center integrals $(\mu \nu \mid P)_{m}$ are defined in eq 15 and originate from the RI factorization of the $4 \mathrm{c}$-CIs given in eq 13 . The expression for $\chi_{P Q}^{0}(i \tau)$ in eq 22 is generic for any RI metric $m\left(\mathbf{r}, \mathbf{r}^{\prime}\right)$. The evaluation of $\chi_{P Q}^{0}(i \tau)$ is the computationally most expensive step and scales $O\left(N^{4}\right)$ with the conventional Coulomb metric (eq 18). However, when employing a local $\mathrm{RI}$ metric, the three-center tensors $(\mu \nu \mid P)_{m}$ vanish unless the Gaussian functions $\phi_{\mu}, \phi_{\nu}$, and $\varphi_{P}$ are centered on nearby atoms, which is illustrated in Figure 2. In this work, we use the local truncated Coulomb metric $m_{r c}$ defined in eq 19. The computational complexity for the evaluation of $\chi_{P Q}^{0}(i \tau)$ reduces with a local metric to $O\left(N^{2}\right)$.

A detailed analysis of the computational complexity of eq 22 is shown in Figure 3. First, the multiplication of the three-center integrals with the Green's function $G$ is computed, which yields the tensors $X$ and $Y$. The evaluation of $X$ and $Y$ scales cubically with a nonlocal metric but only quadratically with a local metric. The subsequent tensor contraction of $X$ and $Y$ is a step of $O\left(N^{4}\right)$ complexity with a nonlocal metric, which is reduced to $O\left(N^{2}\right)$ with the local variant. The $O\left(N^{2}\right)$ scaling behavior in Figure $3 \mathrm{~b}$ can be understood as follows: For computing a single matrix element $\chi_{P Q}^{0}$ with a local metric, only a small $O\left(N^{0}\right)$-scaling number of $\sigma$ indices (spatially close to $P$ ) and $\mu$ indices (spatially close to $Q)$ need to be taken into account. Since the number of $P Q$ pairs increases as $O\left(N^{2}\right)$, we end up with a final scaling of $O\left(N^{2}\right)$ for the whole matrix $\chi_{P Q}^{0}$.

We include the matrix elements $M_{P Q}$ from eq 14 in $\tilde{\chi}^{0}(i \tau)$,

$$
\tilde{\chi}^{0}(i \tau)=\mathbf{M}^{-1} \chi^{0}(i \tau) \mathbf{M}^{-1}
$$

The polarizability $\tilde{\chi}^{0}(i \tau)$ is transformed to imaginary frequency via a cosine transform, and the symmetric dielectric function $\boldsymbol{\epsilon}(i \omega)$ is computed by

$$
\boldsymbol{\epsilon}(i \omega)=1-\mathbf{L}^{\mathrm{T}} \tilde{\chi}^{0}(i \omega) \mathbf{L}
$$

where $\mathbf{L}$ denotes the Cholesky decomposition of the Coulomb matrix $\mathbf{V}$ from eq 16 ,

$$
\mathbf{V}=\mathbf{L L}^{\mathrm{T}}
$$

The screened Coulomb interaction $W(i \omega)=\epsilon^{-1}(i \omega) V=V+$ $W^{c}(i \omega)$ is split into the bare Coulomb interaction and the correlation contribution, and the latter is obtained as 
(a) Nonlocal RI metric ( $m=$ Coulomb)

$\begin{array}{cc}X_{\mu \sigma P}=\sum_{\lambda}(\underline{\lambda \sigma} \mid \underline{P})_{m} G_{\underline{\mu \lambda}}(i \tau) & \underline{\mathcal{O}\left(N^{3}\right)} \\ Y_{\mu \sigma Q}=\sum_{\nu}(\mu \underline{\underline{\nu}} \underline{\underline{Q}})_{m} G_{\underline{\nu \sigma}}(-i \tau) & \underline{\mathcal{O}\left(N^{3}\right)} \\ \chi_{P Q}^{0}(i \tau)=-i \sum_{\mu \sigma} X_{\mu \sigma P}{ }^{Y} \underline{\mu \sigma Q} & \underline{\mathcal{O}\left(N^{4}\right)}\end{array}$

(b) Local RI metric ( $m=$ overlap or truncated Coulomb)

$$
\begin{aligned}
& X_{\mu \sigma P}=\sum_{\lambda}(\underline{\lambda} \sigma \mid P)_{m} G_{\mu \lambda}(i \tau) \quad \underline{\mathcal{O}\left(N^{2}\right)} \\
& Y_{\mu \sigma Q}=\sum_{\nu}(\mu \underline{\nu} \mid Q)_{m} G_{\underline{\nu \sigma}}(-i \tau) \quad \underline{\mathcal{O}\left(N^{2}\right.} \\
& \chi_{P Q}^{0}(i \tau)=-i \sum_{\mu \sigma} X_{\mu \sigma \underline{P}} Y_{\mu \sigma \underline{Q}}
\end{aligned}
$$

Legend: - Green indices (e.g. $\lambda \sigma$ ) are sparse

Figure 3. Scaling of the imaginary-time density response computation in a localized basis (eq 22) together with (a) a nonlocal and (b) a local $\mathrm{RI}$ metric. $\chi_{P Q}^{0}(i \tau)$ is computed in two steps. ${ }^{27}$ First the two tensors $X$ and $Y$ are computed followed by a tensor contraction. The green color indicates sparse indices. The sparse index pair, e.g., $\lambda \sigma$, or sparse index triple, e.g., $\lambda \sigma P$, has together a scaling of $O(N)$. Underlined indices contribute to the scaling.

$$
\mathbf{W}^{\mathrm{c}}(i \omega)=\mathbf{L}\left[\boldsymbol{\epsilon}^{-1}(i \omega)-1\right] \mathbf{L}^{\mathrm{T}}
$$

where the symmetric, positive definite $\boldsymbol{\epsilon}(i \omega)$ is inverted efficiently by Cholesky decomposition.

A cosine transform converts $W^{\mathcal{C}}(i \omega)$ (eq 26) back to the imaginary time domain. Computing the quasiparticle energy for an orbital $\psi_{n}$ requires the corresponding diagonal matrix element of the self-energy,

$$
\Sigma_{n}(i \tau)=\left\langle\psi_{n}|\Sigma(i \tau)| \psi_{n}\right\rangle=: \Sigma_{n}^{x}+\Sigma_{n}^{c}(i \tau)
$$

Its correlation part is obtained as

$$
\Sigma_{n}^{\mathrm{c}}(i \tau)=i \sum_{\nu P} \sum_{\mu} G_{\mu \nu}(i \tau)(n \mu \mid P)_{m} \sum_{Q} \tilde{W}_{P Q}^{\mathrm{c}}(i \tau)(Q \mid \nu n)_{m}
$$

where $\tilde{\mathbf{W}}^{\mathfrak{c}}(i \tau)=\mathbf{M}^{-1} \mathbf{W}^{\mathcal{c}}(i \tau) \mathbf{M}^{-1}$, and the exchange part is computed as

$$
\Sigma_{n}^{\mathrm{x}}=-\sum_{\nu P} \sum_{\mu} D_{\mu \nu}(n \mu \mid P)_{m} \sum_{Q} \tilde{V}_{P Q}(Q \mid \nu n)_{m}
$$

where

$$
D_{\mu \nu}=\sum_{n}^{\mathrm{occ}} C_{n \mu} C_{n \nu}, \quad \tilde{\mathbf{v}}=\mathbf{M}^{-1} \mathbf{V} \mathbf{M}^{-1}
$$

To compute quasiparticle energies, $\Sigma_{n}^{c}(i \tau)$ is transformed to imaginary frequencies by a sine and cosine transform. ${ }^{56}$ The selfenergy is evaluated on the real frequency axis $\Sigma_{n}^{\mathrm{c}}(\varepsilon)$ by analytic continuation of $\Sigma_{n}^{c}(i \omega){ }^{15,25,43,56}$ The $G_{0} W_{0}$ energies $\varepsilon_{n}^{G_{0} W_{0}}$ are obtained by solving the $\mathrm{QP}$ equation

$$
\varepsilon_{n}^{G_{0} W_{0}}=\varepsilon_{n}+\Sigma_{n}^{\mathrm{x}}+\operatorname{Re} \Sigma_{n}^{\mathrm{c}}\left(\varepsilon_{n}^{G_{0} W_{0}}\right)-v_{n}^{\mathrm{xc}}
$$

iteratively for $\varepsilon_{n}^{G_{0} W_{0}}$ via Newton-Raphson.

The calculation of the polarizability $\chi_{P Q}^{0}(i \omega)$ (eq 22$)$ remains the computational bottleneck at $O\left(N^{2}\right)$ complexity, even for the largest systems studied in this work. The subsequent steps in eqs 23-26 scale cubically but have a much smaller computational prefactor. The calculation of the correlation self-energy from eq 28 scales as $O\left(N^{2}\right)$ for every QP level $n$ and is generally computationally less demanding than the calculation of $\chi_{P Q}^{0}(i \tau)$.

4.2. Tracing back Four-Center Coulomb Integrals and RI Factorizations. While the full derivation of the algorithm presented in Section 4.1 is too lengthy, we demonstrate in the following that the three-center integrals $(\mu \nu \mid P)_{m}$ and the metric matrix $\mathbf{M}$ originate indeed from the RI factorization of the $4 \mathrm{c}$ CIs introduced in eq 13 . We will rationalize that the $4 \mathrm{c}$-CIs can be fully recovered with the consequence that our algorithm is exact in the limit of a complete RI basis set.

For the exchange part of the self-energy, the 4c-CIs can be directly obtained by inserting eq 30 into eq 29 and using eq 13, which yields the familiar expression for the exchange selfenergy, ${ }^{25} \sum_{n}^{\mathrm{x}}=-\sum_{i}^{\mathrm{occ}}(\text { nilin })_{\mathrm{RI}}$.

The RI factorization of the $4 c$-CIs is less obvious for the correlation part $\Sigma_{n}^{c}$ of the self-energy and the intermediate steps. We exemplarily show for the matrix elements $W_{P Q}^{c}$ where the $4 \mathrm{c}$ CIs occur. To this end, we use the Taylor expansion $(1-x)^{-1}=$ $1+x+x^{2}+\ldots$ to express the inverse of the dielectric function from eq 24 as

$$
\boldsymbol{\epsilon}^{-1}(i \omega)=1+\mathbf{L}^{\mathrm{T}} \tilde{\chi}^{0}(i \omega) \mathbf{L}+\left(\mathbf{L}^{\mathrm{T}} \tilde{\chi}^{0}(i \omega) \mathbf{L}\right)^{2}+\ldots
$$

We can then rewrite eq 26 as

$$
\mathbf{W}^{\mathrm{c}}(i \omega)=\mathbf{L}\left[\mathbf{L}^{\mathrm{T}} \tilde{\chi}^{0}(i \omega) \mathbf{L}+\left(\mathbf{L}^{\mathrm{T}} \tilde{\chi}^{0}(i \omega) \mathbf{L}\right)^{2}+\ldots\right] \mathbf{L}^{\mathrm{T}}
$$

After inserting eqs 23 and 25 into eq 33, we obtain

$$
\begin{aligned}
\mathbf{W}^{\mathrm{c}}(i \omega) & =\mathbf{V M}^{-1} \chi^{0}(i \omega) \mathbf{M}^{-1} \mathbf{V} \\
& +\mathbf{V} \mathbf{M}^{-1} \chi^{0}(i \omega) \mathbf{M}^{-1} \mathbf{V} \mathbf{M}^{-1} \chi^{0}(i \omega) \mathbf{M}^{-1} \mathbf{V}+\ldots
\end{aligned}
$$

With eq 22, we recover the RI expression (eq 13) in the quadratic term:

$$
\begin{aligned}
& {\left[\chi^{0}(i \omega) \mathbf{M}^{-1} \mathbf{V M}^{-1} \chi^{0}(i \omega)\right]_{P Q} } \\
&=- \sum_{R S T U} \sum_{\mu \sigma \lambda \nu} \sum_{\mu \sigma \lambda \nu} \operatorname{FT}\left[G_{\mu \lambda}(i \tau) G_{\nu \sigma}(-i \tau)\right](i \omega)(\lambda \sigma \mid P)_{m}(\mu \nu \mid R)_{m} \\
& \times M_{R S}^{-1} V_{S T} M_{T U}^{-1} \operatorname{FT}\left[G_{\overline{\mu \lambda}}(i \tau) G_{\overline{\nu \sigma}}(-i \tau)\right](i \omega)(\overline{\lambda \sigma} \mid U)_{m}(\overline{\mu \nu} \mid Q)_{m} \\
&=-\sum_{\mu \sigma \lambda \nu} \sum_{\mu \sigma \lambda \nu} \operatorname{FT}\left[G_{\mu \lambda}(i \tau) G_{\nu \sigma}(-i \tau)\right](i \omega)(\lambda \sigma \mid P)_{m}(\mu \nu \mid \overline{\lambda \sigma})_{\mathrm{RI}} \\
& \quad \times \operatorname{FT}\left[G_{\overline{\mu \lambda}}(i \tau) G_{\overline{\nu \sigma}}(-i \tau)\right](i \omega)(\overline{\mu \nu} \mid Q)_{m}
\end{aligned}
$$

The RI expression (eq 13) can be found in a similar fashion for all higher orders in $W_{P Q}^{c}$ and ultimately also for the expression of the self-energy in eq 28.

\section{IMPLEMENTATION DETAILS}

We have implemented the low-scaling $G W$ algorithm outlined in Section 4.1 in the open-source software package CP2K, ${ }^{90}$ which is available from github. ${ }^{91}$ The parallelization of the algorithm is mostly based on the standard message passing interface (MPI). OpenMP threading in a hybrid MPI+OpenMP approach is also supported. All steps of the algorithm have been optimized for massively parallel executation on more than 10000 CPU cores. Most optimization efforts were dedicated to the computationally most expensive step, the calculation of $\chi_{P Q}^{0}(i \omega)$, using the 
concepts outlined in ref 27 and the DBCSR library for sparse matrix-tensor operations. ${ }^{92} \mathrm{DBCSR}$ is also employed for sparse matrix-matrix operations in eqs 28 and 29.

The proper choice and optimization of the imaginary-time and imaginary-frequency grids are crucial for computational efficiency and accuracy. We employ the minimax time $\left\{\tau_{j}\right\}_{j=1}^{N}$ and frequency $\left\{\omega_{k}\right\}_{k=1}^{N}$ grids with $N$ grid points as pioneered by Kaltak et al. ${ }^{93}$ and Liu et al. ${ }^{56}$ For minimax, the a priori known analytical structure of $\chi, W$, and $\Sigma$ is used to construct grids that minimize the $L^{\infty}$ norm of the error between the exact integration and the numerical integration. Following this procedure, optimal grids can be constructed for the Fourier transforms ${ }^{56,93}$ of the respective functions $f$,

$$
\begin{aligned}
& f\left(i \omega_{k}\right)=\sum_{j=1}^{N} \gamma_{k j} \exp \left(i \omega_{k} \tau_{j}\right) f\left(i \tau_{j}\right) \\
& f\left(i \tau_{j}\right)=\sum_{k=1}^{N} \xi_{j k} \exp \left(-i \tau_{j} \omega_{k}\right) f\left(i \omega_{k}\right)
\end{aligned}
$$

For simplicity, we compute the weights $\gamma_{k j}$ and $\xi_{j k}$ during the program execution from $L^{2}$ minimization. ${ }^{93}$

Minimax grids are constructed by the Remez algorithm, which requires higher numerical precision than the standard double precision used in electronic-structure calculations. The minimax grids are therefore not optimized during run-time, but computed with quadruple precision and pretabulated. ${ }^{94}$ For details on generating minimax grids, we refer to the comprehensive literature. $^{56,93}$ Note that minimax grids were recently also developed for finite-temperature $G W .^{95}$

In our previous work, ${ }^{19}$ we employed 12 minimax points. To achieve higher accuracy, we have now computed minimax grids with $26,28,30,32$, and 34 points in imaginary time and imaginary frequency for different ranges. ${ }^{93}$ These grids are freely available on github ${ }^{91}$ for usage with other codes implementing the space-time method. As we demonstrate in Section 7, benchmark accuracy is already obtained with 30 minimax points. Since the convergence of the Remez algorithm is increasingly difficult with the number of points, the generation of grids with more than 34 points has not been attempted.

\section{COMPUTATIONAL DETAILS}

The low-scaling GW calculations are performed with the program package $\mathrm{CP} 2 \mathrm{~K}^{90}$, and reference calculations are carried out with the program package FHI-aims. ${ }^{96}$ The input and output files of these calculations are available from the Novel Materials Discovery (NOMAD) repository. ${ }^{97}$

6.1. Low-Scaling GW Calculations Using CP2K. We perform $G_{0} W_{0}$ calculations with the low-scaling algorithm on the $G W 100$ benchmark set (Section 7) and $G_{0} W_{0}$ as well as evGW calculations on phosphorene nanosheets (Sections 8-10). All $G W$ calculations start from all-electron DFT calculations using the Gaussian and augmented plane-waves scheme (GAPW) ${ }^{98}$ and the Perdew-Burke-Ernzerhof (PBE) ${ }^{99}$ exchange-correlation functional. We use the RI with the truncated Coulomb metric with a truncation radius of $r_{\mathrm{c}}=3 \AA$, unless otherwise noted. The self-energy is analytically continued from the imaginary to the real-frequency domain using a Padé model $^{15,56,100}$ with 16 parameters.

For the GW100 benchmark calculations, we use the def2QZVP ${ }^{101}$ basis set as the primary basis set and def2-TZVPPDRIFIT $^{102}$ as the auxiliary basis set. We employ minimax grids with $N=30$ time and frequency minimax points for the GW100 study, unless otherwise stated.

The molecular geometries of the phosphorene nanosheets are obtained as follows: We relax the unit cell of free-standing phosphorene using PBE-D3, ${ }^{99,103}$ Goedecker-Teter-Hutter pseudopotentials, ${ }^{104}$ and a TZVP-MOLOPT basis set ${ }^{105}$ using an $8 \times 6 k$-point mesh. Then, an $L \times L(L \in \mathbb{N})$ supercell is formed, periodic boundary conditions are removed, and dangling bonds are saturated by hydrogen atoms. The hydrogen atoms are relaxed with PBE-D3 while keeping the phosphorus atoms fixed.

For the $G W$ calculations on phosphorene nanosheets, we employ the all-electron aug-cc-pVDZ basis sets ${ }^{106-108}$ and the RI basis set aug-cc-pVDZ-RIFIT. ${ }^{89,102,109}$ The lowest exponents of the RI basis set have been scaled up for the calculations on the large phosphorene sheets reported in Section 9 to improve the performance; see the SI for details. Minimax grids with 30 time and frequency points are used for the small phosphorene clusters studied in Section 8, while 14 minimax points are used for the large phosphorene sheets. In the sparse matrix-tensor operations from eq 22, we filter atomic tensor blocks conservatively with a Frobenius norm of the atomic blocks of $10^{-15}$. For evG $W_{0}$, we employ 80 occupied and 80 unoccupied $G W$ levels in the self-consistency loop. For levels outside this range, a constant shift in the evG $W_{0}$ cycle has been applied.

With these settings, we find that the $G_{0} W_{0}$ and evGW HOMO-LUMO gap of large phosphorene sheets (Section 9) is converged within $0.02 \mathrm{eV}$ compared to calculations using a fully converged minimax grid of 30 points and the aug-cc$\mathrm{pVQZ}^{106,108}$ basis set; see the SI for more details. An extrapolation to the complete basis set limit, as often necessary in $G W$, is therefore not required. HOMO-LUMO gaps typically converge faster with respect to basis set size than ionization potentials and affinities, which was demonstrated in, e.g., ref 21 for subsets of medium and large molecules from the GW5000 database. $^{26}$

Additionally, we compute the PBE gap of $2 \mathrm{D}$ periodic phosphorene from GAPW all-electron calculations using the aug-cc-pVTZ basis set ${ }^{106-108}$ and an $8 \times 6 k$-point mesh.

6.2. Reference GW Calculations with Contour Deformation Using FHI-aims. We perform reference DFT calculations with the PBE functional for all phosphorene nanosheets and $G_{0} W_{0} @ \mathrm{PBE}$ calculations for the smaller phosphorene nanosheets up to 180 atoms using the FHI-aims program package. ${ }^{96} \mathrm{FHI}$-aims is a native all-electron code based on numeric atomic-centered orbitals (NAOs). For direct comparison with the low-scaling calculations, we employ also the aug-cc-pVDZ Gaussian basis sets, which can be considered as a special case of an NAO and which are treated numerically in FHI-aims. The auxiliary basis sets are constructed "on the fly" by forming product pairs of primary basis functions and subsequent removal of linear dependencies as described in ref 46.

The GW calculations are performed with the contour deformation implementation ${ }^{11}$ in FHI-aims, unless otherwise noted. As for the low-scaling $\mathrm{CP} 2 \mathrm{~K}$ calculations, the QP equations are always solved iteratively. In addition to computing the QP energies for the phosphorene nanosheets, we also compute the self-energy matrix elements for a small phosphorene cluster with 24 atoms comparing contour deformation and analytic continuation. ${ }^{46}$ For the latter, we use the Padé approximation with 16 parameters, as in the CP2K calculations. Both methods, contour deformation and analytic continuation, require the computation of integrals over the imaginary 
frequency axis, for which we employ a modified GaussLegendre grid ${ }^{46}$ with 200 grid points. For the Padé model, the same set of grid points $\{i \omega\}$ is used to calculate $\Sigma_{n}^{c}(i \omega)$.

Using the same basis set, the DFT-PBE gaps of the phosphorene sheets agree within $1 \mathrm{meV}$ between $\mathrm{CP} 2 \mathrm{~K}$ and FHI-aims and the $G_{0} W_{0}$ gaps within $20 \mathrm{meV}$; see Table II, SI.

\section{GW100 BENCHMARK: ACCURACY OF FRONTIER ORBITALS}

In the following, we assess the accuracy of the low-scaling $G W$ algorithm for HOMO and LUMO QP energies of molecules from the GW100 benchmark set. ${ }^{15}$ We carefully study their convergence with the minimax integration grid size, the RI basis set size, and the truncation radius used for the RI-tC metric.

7.1. Data Set and Reference Values. The GW100 benchmark set contains HOMO and LUMO energies of 100 small molecules featuring a variety of elements from the periodic table. We exclude the multisolution cases $\mathrm{BN}, \mathrm{BeO}, \mathrm{MgO}, \mathrm{O}_{3}$, and $\mathrm{CuCN}$ from computing the MAD of the HOMOs for the following reasons. First, the real self-energy matrix elements of these molecules exhibit poles in the frequency region of the quasiparticle, leading to at least two different solutions with similar spectral weights. ${ }^{15}$ Different codes might find equally valid solutions, and one should rather compare the self-energy matrix elements, as done in ref 15 . Second, 128 Padé parameters are necessary to resolve these poles. ${ }^{15}$ This implies that $\Sigma(i \omega)$ must be computed on a frequency grid of at least 128 points, which is far beyond the size of the currently available minimax grids. All 100 molecules are included for the MAD of the LUMO.

We use the $G_{0} W_{0} @ P B E$ results from FHI-aims reported in the original GW100 work ${ }^{15}$ as a reference. The FHI-aims results from ref 15 were computed with analytical continuation using the Padé model approximation with 16 parameters, as in our approach. The analytic-continuation results from FHI-aims are of high numerical quality for frontier orbitals, matching the results from a fully analytic evaluation of the self-energy within a few meV, as shown for a GW100 subset in ref 15 . Our goal is to assess the numerical accuracy of the algorithm for a given primary basis set. We therefore compare the data directly at the def2-QZVP level instead of basis-set extrapolated results.

7.2. Convergence of the Minimax Grid. The convergence of the $G_{0} W_{0} @ P B E$ QP energies with respect to to the size of our generated minimax grids is reported in Table 1. Except for different minimax parameters, the settings given in Section 6.1 were used. The MADs with respect to the GW100 reference results decrease quickly with the grid size. Already for 28 minimax points, we observe an MAD of $<10 \mathrm{meV}$ for both HOMOs and LUMOs. The accuracy saturates at 30 minimax points with an MAD of $7 \mathrm{meV}$ for HOMOs and $6 \mathrm{meV}$ for LUMOs. The gain of accuracy when employing even larger grids with 32 and 34 points is marginal. Therefore, we set the minimax grid with 30 points as default for benchmark studies with the low-scaling algorithm.

The low-scaling $G W$ algorithm reported in ref 56, which is the PAW variant of the space-time method, reaches high accuracy already for smaller minimax grids. Liu et al. ${ }^{56}$ showed that 20 time and frequency points were sufficient to reach convergence within $10 \mathrm{meV}$. As shown in Table 1, the MAD is still larger than $20 \mathrm{meV}$ with the same grid size in our scheme. The different convergence behavior is probably due to the different treatment of the core electrons. The low-scaling PAW-GW schemes does not treat the core electrons explicitly, which reduces the
Table 1. Convergence of HOMO and LUMO Energies of the GW100 Benchmark Set Computed with the Low-Scaling Algorithm at the $G_{0} W_{0} @ P B E$ Level as a Function of the Number of Minimax Points $N^{a}$

\begin{tabular}{|c|c|c|c|c|c|c|}
\hline \multirow[b]{2}{*}{$N$} & \multicolumn{2}{|c|}{ MAD (eV) } & \multicolumn{2}{|c|}{$\mathrm{MAD} \leq 0.01 \mathrm{eV}$} & \multicolumn{2}{|c|}{$\mathrm{MAD} \leq 0.02 \mathrm{eV}$} \\
\hline & HOMOs & LUMOs & HOMOs & LUMOs & HOMOs & LUMOs \\
\hline 10 & 0.098 & 0.046 & 24 & 26 & 36 & 41 \\
\hline 20 & 0.025 & 0.013 & 32 & 77 & 66 & 96 \\
\hline 26 & 0.014 & 0.009 & 75 & 92 & 84 & 98 \\
\hline 28 & 0.009 & 0.007 & 81 & 94 & 89 & 97 \\
\hline 30 & 0.007 & 0.006 & 87 & 93 & 92 & 98 \\
\hline 32 & 0.007 & 0.005 & 88 & 94 & 91 & 99 \\
\hline 34 & 0.007 & 0.005 & 92 & 95 & 93 & 98 \\
\hline
\end{tabular}

${ }^{a}$ Listed are the mean absolute deviations (MADs) with respect to the FHI-aims reference values from ref 15 (16-parameter Padé model, def2-QZVP) and the number of excitations (out of 95 for the HOMO and out of 100 for LUMO) with MADs $\leq 0.01 \mathrm{eV}$ and $\leq 0.02 \mathrm{eV}$.

minimax range ${ }^{93}$ compared to our all-electron scheme. With smaller minimax ranges, fewer grid points are generally needed to obtain the same accuracy.

7.3. Convergence of RI Basis Sets and the Truncation Radius. The other two parameters, which influence the accuracy of the low-scaling algorithm, are the RI basis set size and the Coulomb cutoff radius for the RI-tC metric. Both parameters are in principle interdependent since the cutoff radius controls if the metric is more "overlap-like" or rather resembles the conventional Coulomb metric, which requires smaller RI basis set sizes as discussed in Section 3.2.

Figure 4a,b shows the MAD between the low-scaling $G W$ algorithm and the FHI-aims results for the GW100 test set data as a function of the RI basis set size for the HOMO and LUMO, respectively. We study the $\mathrm{RI}$ basis set convergence for two cutoff values, $r_{\mathrm{c}}=1 \AA$ and $r_{\mathrm{c}}=3 \AA$. We observe a more consistent convergence behavior when using the larger cutoff $r_{\mathrm{c}}=3 \AA$. For $r_{\mathrm{c}}=1 \AA$, the smallest MAD $(10 \mathrm{meV})$ is obtained with the def2TZVPPD-RIFIT basis. The accuracy becomes worse for larger RI basis sets which might be related to ill-conditioning problems. The truncation at $r_{\mathrm{c}}=3 \AA$ yields a higher accuracy than $r_{\mathrm{c}}=1 \AA$ for all $\mathrm{RI}$ basis sets that have been tested. The MAD is well below $10 \mathrm{meV}$ for def2-TZVPPD-RIFIT and the next larger RI basis set.

In Figure 4c,d, we employ def2-TZVPPD-RIFIT as the RI basis and vary the Coulomb cutoff radius used in RI-tC. For $r_{c}=0 \AA$, the RI metric is equivalent to the overlap metric, and we obtain an MAD of $\sim 30 \mathrm{meV}$ for the HOMO, which is close to the $35 \mathrm{meV}$ deviation reported in our previous work ${ }^{19}$ for the low-scaling algorithm with the overlap metric. The accuracy improves when increasing the Coulomb cutoff radius saturating at radii $r_{\mathrm{c}} \geq 2 \AA$. This observation and the rapid convergence of the RI basis set with $r_{\mathrm{c}} \geq 3 \AA$ in Figure $4 \mathrm{a}, \mathrm{b}$ imply that the attractive features of the conventional Coulomb metric are already largely restored at truncation radii between 2 and $3 \AA$. We choose $r_{c}=3 \AA$ as a safe setting for our low-scaling calculations.

7.4. Benchmark with Converged Settings. We now compare the GW100 results obtained with the settings given in Section 6.1, i.e., the converged settings (30 minimax points, def2-TZVPPD-RIFIT, $r_{\mathrm{c}}=3 \AA$ ) to the FHI-aims reference data. The number of molecules with a given absolute deviation from the reference data are shown in Figure 5 (see Table I, SI, for the raw data). We find that 87 out of $95 \mathrm{HOMO}$ energies and 93 out 

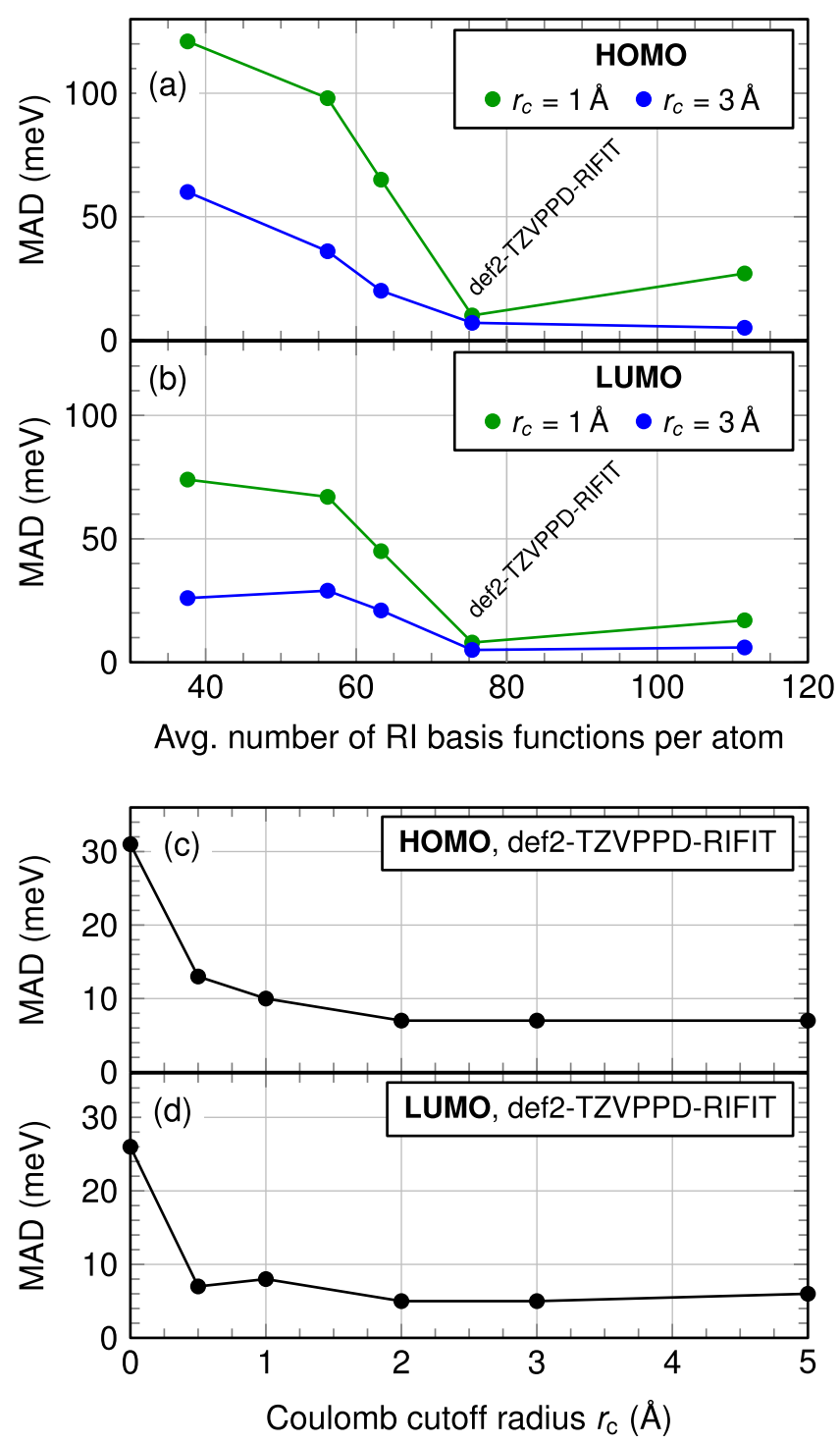

Figure 4. Convergence of HOMO and LUMO energies of the GW100 benchmark set computed with the low-scaling algorithm at the $G_{0} W_{0} @$ PBE level with respect to (a, b) the RI basis set size and (c, d) the truncation radius from eq 19. Presented are the mean absolute deviations (MADs) with respect to the FHI-aims reference data ${ }^{15}$ (16parameter Padé model, def2-QZVP). For (a) and (b), we employ the RI basis sets def2-SVP-RIFIT, def2-TZVP-RIFIT, def2-TZVPP-RIFIT, def2-TZVPPD-RIFIT, and def2-QZVPP-RIFIT. ${ }^{102}$ In (c) and (d), the def2-TZVPPD-RIFIT basis is used as the RI basis set.

of 100 LUMO energies agree with the FHI-aims reference ${ }^{15}$ within $10 \mathrm{meV}$; see also Table 1 . Only three excitations for the HOMO and two excitations for the LUMO differ by more than $20 \mathrm{meV}$ from the reference, and the maximum deviation is 50 $\mathrm{meV}$.

Compared to our old implementation with the overlap metric, ${ }^{19}$ the accuracy is significantly improved. The MAD is reduced from 35 to $7 \mathrm{meV}$ for the $\mathrm{HOMO}$ and from 27 to $6 \mathrm{meV}$ for the LUMO. The MAD is now in the range that was reported for the FHI-aims reference data and the fully analytic Turbomole results $(3 \mathrm{meV}$ for the HOMO and $6 \mathrm{meV}$ for the LUMO).$^{15}$ The RI-tC scheme and the new minimax grids also improve the reliability of the low-scaling algorithm. The number of outliers is reduced to zero. In our previous work, ${ }^{19}$ we observed 9 energies with deviations $\geq 60 \mathrm{meV}$ for the HOMO (a)

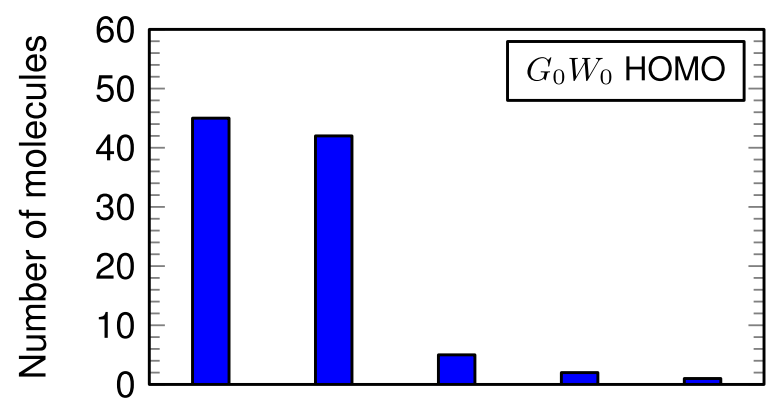

(b)

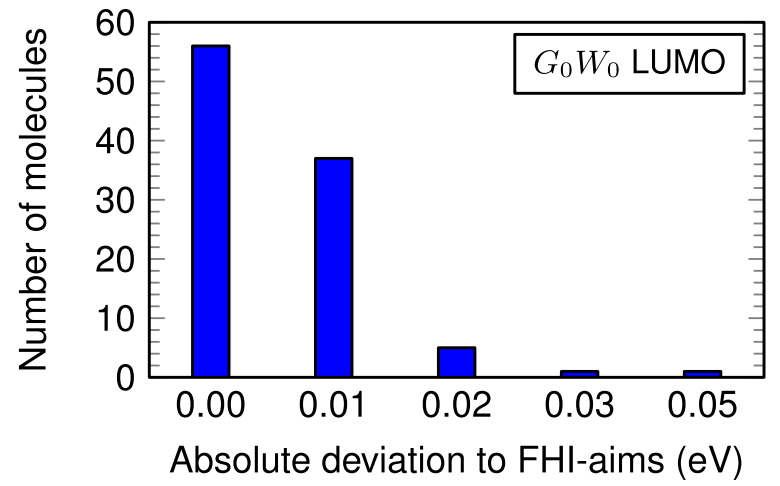

Figure 5. GW100 benchmark of the $G_{0} W_{0} @ P B E$ energies computed with the low-scaling algorithm and the settings given in Section 6.1 for (a) HOMOs and (b) LUMOs. Shown are the number of molecules with a given absolute deviation to the FHI-aims values from ref 15 (16parameter Padé, def2-QZVP); see SI for raw data.

and 7 for the LUMO, including a couple of extreme cases with errors of 0.7 and $2.2 \mathrm{eV}$.

\section{ACCURACY FOR SEMICORE AND UNBOUND STATES}

The structure of the self-energy matrix elements $\Sigma_{n}^{c}(\omega)$ is typically featureless around the QP solutions for the HOMO and LUMO. ${ }^{2,11,15}$ Achieving benchmark accuracy is thus easier for states close to the Fermi level. A more challenging test for our low-scaling algorithm are semicore, deep valence and unbound states. In Figure 6a, we report all $G_{0} W_{0}$ QP energies within a 20 $\mathrm{eV}$ distance to either HOMO or LUMO for a small phosphorene nanosheet cluster $\left(\mathrm{H}_{10} \mathrm{P}_{14}\right)$. The cluster is shown as the inlet in Figure $6 c$, and its geometry is reported in the SI. The results are compared to QP energies computed with the highly accurate contour deformation technique (CD) implemented in FHIaims. ${ }^{11}$ We have previously shown that the $\mathrm{CD}$ technique with the settings described in Section 6.2 yields without exception the same numerical accuracy as the fully analytic evaluation of the self-energy, including the difficult case of deep core states. ${ }^{11}$ By design, the $C D$ techniques are more accurate than the analytic continuation. We set thus the CD results from FHI-aims as the reference for our benchmark of semicore and unbound states.

We find that all frontier orbitals in the frequency range $\mathrm{HOMO}-2 \mathrm{eV}$ and $\mathrm{LUMO}+2 \mathrm{eV}$ deviate by at most $0.02 \mathrm{eV}$, comparing the $\mathrm{CD}$ results with the energy value of the lowscaling $G W$ algorithm introduced in this work; see Figure 6a. The deviation increases with increasing distance from the Fermi level. However, the error is for all levels between -20 and $20 \mathrm{eV}$ below $0.10 \mathrm{eV}$.

The increasing deviation is attributed to the analytic continuation technique, which is employed in our low-scaling $G W$ algorithm. In the final step of the algorithm, the self-energy 

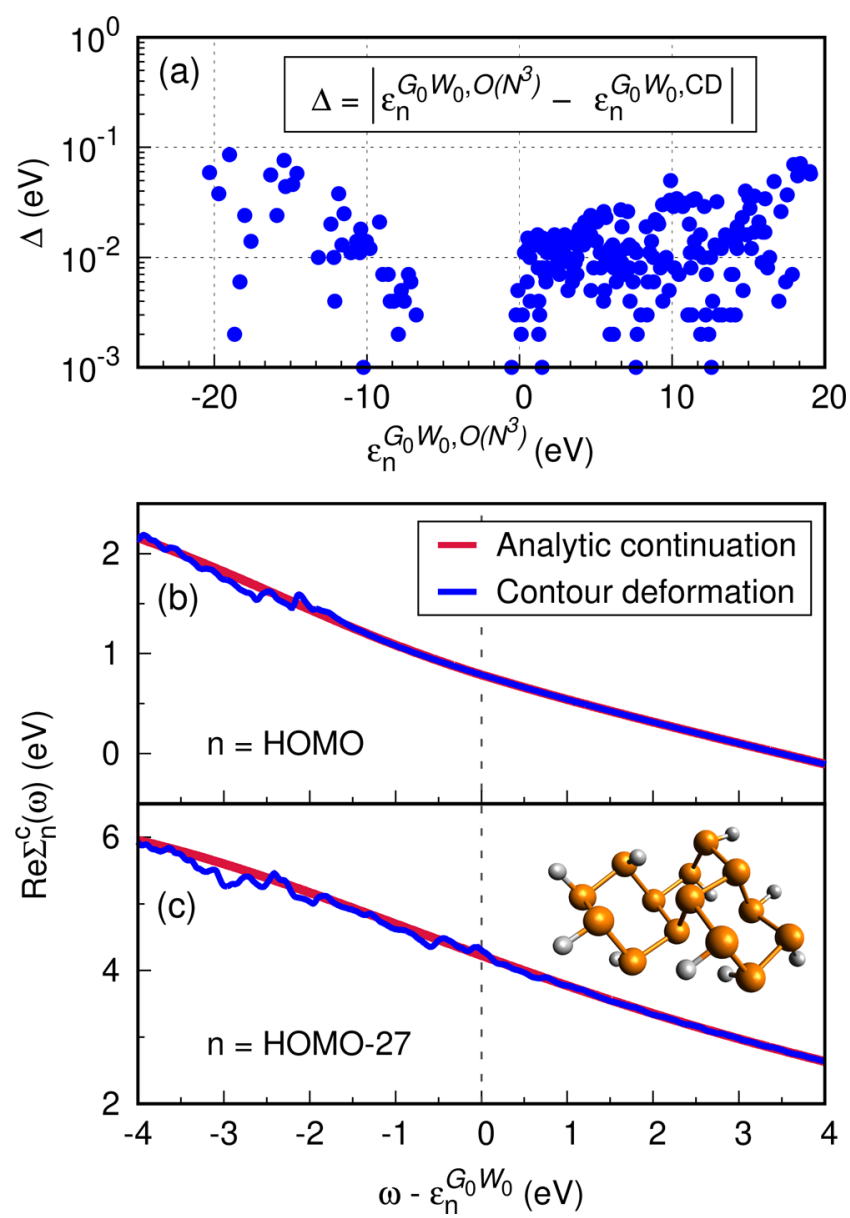

Figure 6. $G_{0} W_{0}$ quasiparticle energies of the phosphorene nanosheet $\mathrm{H}_{10} \mathrm{P}_{14}$ including all states between -20 to $20 \mathrm{eV}$. (a) Absolute deviation $\Delta:=\left|\varepsilon_{n}^{G_{0} W_{0}, O\left(N^{3}\right)}-\varepsilon_{n}^{G_{0} W_{0}, C D}\right|$ of the $G_{0} W_{0}$ energies computed with the low-scaling $\left(O\left(N^{3}\right)\right)$ algorithm implemented in $\mathrm{CP} 2 \mathrm{~K}$ with RI-tC (this work) to the contour deformation (CD) implementation in FHI-aims. ${ }^{11}$ (b, c) Real part of the correlation self-energy $\Sigma^{c}(\omega)$ computed with FHI-aims comparing contour deformation and analytic continuation. Diagonal matrix elements $\operatorname{Re} \Sigma_{n}^{c}(\omega)=\left\langle\psi_{n}\left|\operatorname{Re} \Sigma^{c}(\omega)\right| \psi_{n}\right\rangle$ for (b) the HOMO and (c) the semicore state HOMO-27. Note that the "ripples" in the self-energy in (b) and (c) are broadened, shallow poles.

is analytically continued to the real axis by fitting the matrix elements $\Sigma_{n}^{c}(i \omega)$ to a multipole model. These models are usually flexible enough to describe frontier orbitals, as shown in Figure $6 \mathrm{~b}$. The self-energy is smooth in the frequency region of the HOMO QP energy, and $\Sigma_{\mathrm{HOMO}}^{c}(\omega)$ is perfectly reproduced by the analytic continuation around the HOMO QP energy. For deep valence and semicore states and unbound states, $\Sigma_{n}^{c}$ increasingly acquires features around the QP energy. This is demonstrated for the state HOMO-27 of the phosphorene nanosheet cluster in Figure 6c. The real part of $\Sigma_{c}^{n}(\omega)$ has shallow poles around $\omega=\varepsilon_{n}^{G_{0} W_{0}}$, which are broadened in our $\mathrm{CD}$ calculation. These broadened poles appear as "ripples" in the self-energy. It is practically impossible to reproduce these small pole features with analytic continuation exactly. This is true for canonical $O\left(\mathrm{~N}^{4}\right)$ as well as low-scaling implementations of the analytic continuation.

The acquisition of pole features around the QP energy for states far from the Fermi level is a conceptual problem of
$G_{0} W_{0} @$ PBE. This can be best understood when rewriting the self-energy into its analytic form ${ }^{47}$

$$
\Sigma_{n}^{c}(\omega)=\sum_{m} \sum_{s} \frac{\left\langle\psi_{n} \psi_{m}\left|P_{s}\right| \psi_{m} \psi_{n}\right\rangle}{\omega-\varepsilon_{m}+\left(\Omega_{s}-i \eta\right) \operatorname{sgn}\left(\varepsilon_{\mathrm{F}}-\varepsilon_{m}\right)}
$$

where $m$ runs over all occupied and virtual states and $\eta$ is a broadening parameter. $\Omega_{s}$ are charge neutral excitations and $P_{s}$ the corresponding transition amplitudes. From eq 39 we directly see that the self-energy $\Sigma_{n}^{c}(\omega)$ has real-valued poles (for $\eta \rightarrow 0$ ) at $\varepsilon_{i}-\Omega_{s}$ for occupied states and $\varepsilon_{a}+\Omega_{s}$ for virtual states. As we discussed in detail in ref 12 , these poles give rise to satellite features, which accompany the QP excitation. The neutral excitations $\Omega_{s}$, which are close to eigenvalue differences, are underestimated at the PBE level. For occupied states, the PBE orbital energies $\varepsilon_{n}$ are overestimated, and the poles $\varepsilon_{i}-\Omega_{s}$ are located at too large (too positive) frequencies and are too close to the QP energy. For virtual states, the reasoning is the same, just with reverse sign, i.e., $\varepsilon_{a}+\Omega_{s}$ are at too small frequencies.

The problem that $\varepsilon_{i}-\Omega_{s}$ are located at too positive frequencies gets progressively worse for deep states, since the difference between the PBE eigenvalues and corresponding QP energies increases in absolute terms. This behavior is visible in Figure $6 \mathrm{~b}, \mathrm{c}$ for the calculation with the exact $\mathrm{CD}$ technique. The shallow pole structure is for $\mathrm{HOMO}-27$ in the frequency region of the QP energy, $\omega=\varepsilon_{n}^{G_{0} W_{0}}$, whereas for the HOMO the shallow pole structure is located $2 \mathrm{eV}$ off from the QP energy.

It has been shown that the correct distance between the poles and the QP solution can be restored in an evGW $W_{0}$ scheme, ${ }^{110,111}$ even in the extreme case of deep core excitations. ${ }^{12}$ Since the effect of eigenvalue self-consistency in $G$ is to push the pole structure away from the QP energy, ${ }^{2,12}$ i.e., to more negative and positive frequencies for occupied and unoccupied states, respectively, the self-energy structure is also easier to model by analytic continuation for semicore states and unbound states. We thus expect that the numerical accuracy of our low-scaling algorithm for nonfrontier orbitals is even better than that shown in Figure 6a when using an evGW $W_{0}$ scheme.

\section{HOMO-LUMO GAP OF PHOSPHORENE NANOSHEETS FROM GW}

We apply our low-scaling $G W$ code to finite hyrogen-terminated nanosheets of phosphorene. Phosphorene consists of a single layer of black phosphorus and was first synthesized in 2014. ${ }^{120,121}$ Phosphorene forms an armchair-like vertically buckled structure of $s p^{3}$ bonded phosphorus atoms, as shown in Figure 7a,b. It has attracted vibrant research interest as a twodimensional semiconductor ${ }^{122}$ because of its direct band gap of $\approx 2 \mathrm{eV}$ at the $\Gamma$ point. ${ }^{118,119}$ The band gap can be successively decreased from 2 to $0.3 \mathrm{eV}$ (3D bulk limit) by increasing the number of layers. ${ }^{123}$ This band gap range is ideal for many optoelectronic, photovoltaic, and photocatalytic applications. ${ }^{123}$ Deformation $^{124}$ and twisting of layers ${ }^{125}$ have been also proposed as methods to modify the band gap of phosphorene.

We show in this work that the band gap can be also engineered in the in-plane direction toward values larger than $2 \mathrm{eV}$ by exploiting finite size effects, which has been recently also reported from Quantum Monte Carlo calculations. ${ }^{112}$ We study here rectangular hydrogen-terminated phosphorene sheets of size $L \times L(L \in \mathbb{N})$, where $L$ indicates the repetition of the phosphorene unit cell; see Figure $7 \mathrm{a}, \mathrm{b}$ for a sketch of the molecular geometry. The smallest sheet $(4 \times 4)$ is of size 1.8 


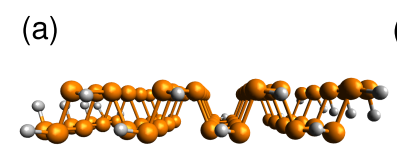

(c)
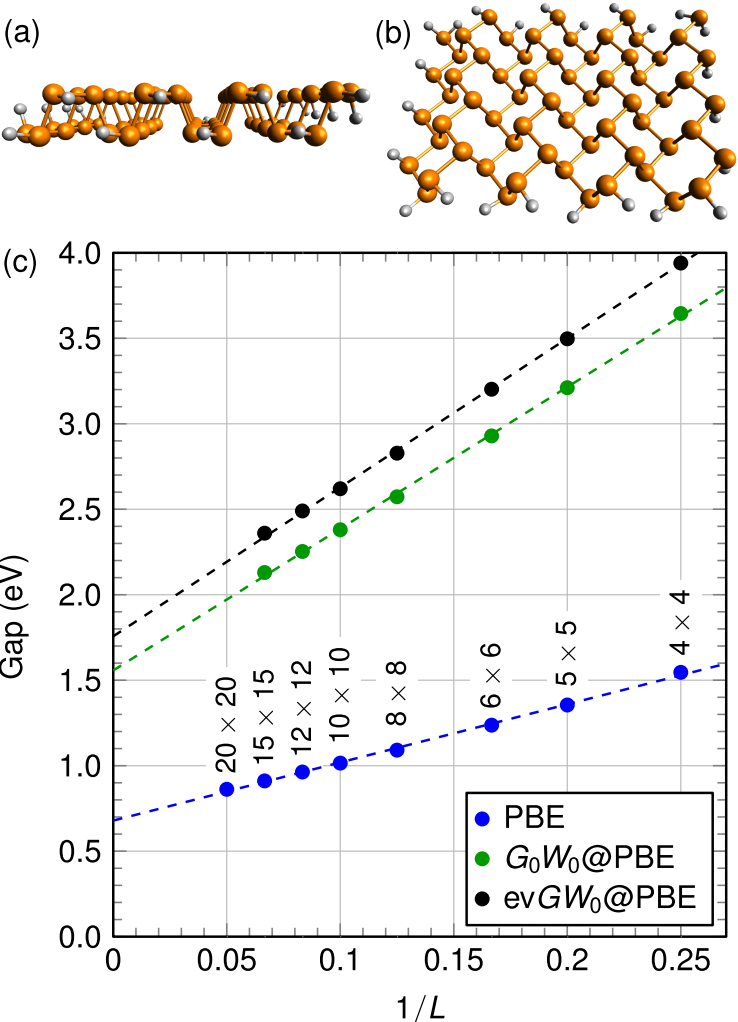
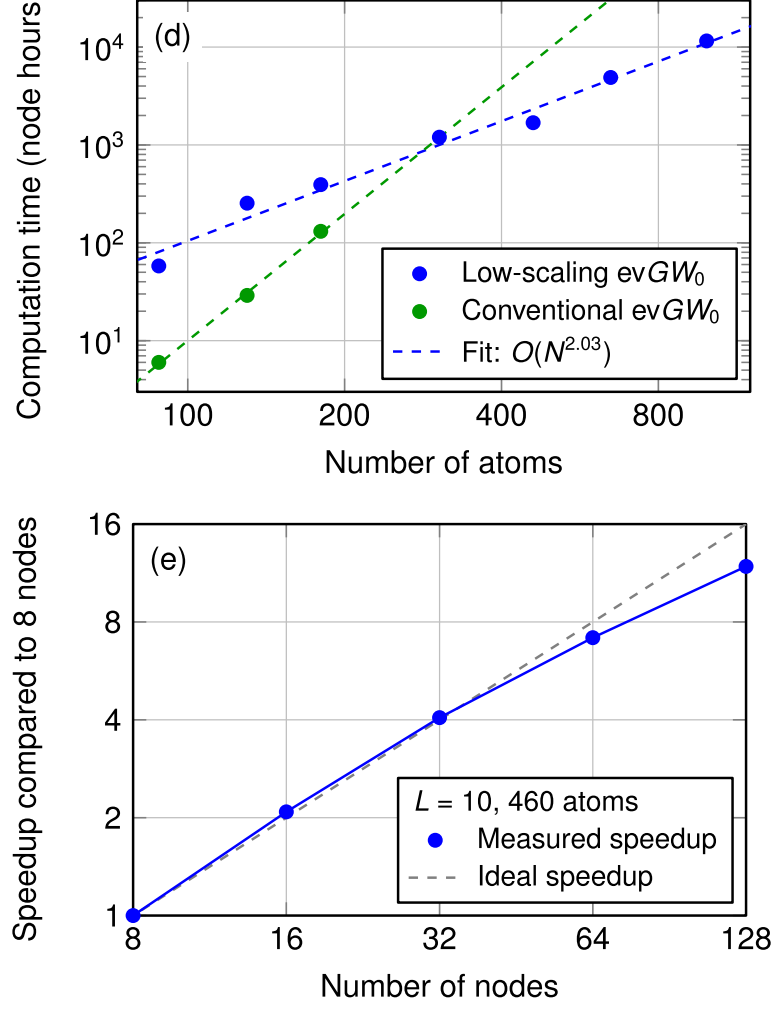

Figure 7. (a) Side and (b) top views of the $4 \times 4$ phosphorene nanosheet. (c) HOMO-LUMO gap of $L \times L$ phosphorene nanosheets computed from DFT-PBE, $G_{0} W_{0} @ P B E$, and evG $W_{0} @ P B E$ as a function of the inverse number $1 / L$ of unit cells along an edge of the $L \times L$ nanosheet. (d) Scaling of the evGW $W_{0}$ execution time with number of atoms for the $L \times L$ phosphorene nanosheets comparing the low-scaling implementation from this work to the conventional implementation ${ }^{25}$ with $O\left(\mathrm{~N}^{4}\right)$ scaling. Dashed lines are two-parameter least-squares fits of the prefactor and exponent. (e) Scaling of the low-scaling $G W$ implementation with respect to the number of computing nodes. Presented are strong scaling measurements for the $10 \times 10$ phosphorene sheet (460 atoms) using the cc-pVDZ basis set. ${ }^{106}$ (Note that aug-cc-pVDZ basis is used in (c) in (d).) The calculations in (c), (d), and (e) have been executed on processors of the type "Skylake Xeon Platinum 8174" (48 processors per node) with 96 GB memory (c and d) and 768 GB memory (e) per node.

$\mathrm{nm} \times 1.3 \mathrm{~nm}$, while the largest $(20 \times 20)$ is of dimension 9.2 $\mathrm{nm} \times 6.7 \mathrm{~nm}$. The progression of the fundamental HOMOLUMO gaps computed from DFT-PBE eigenvalues and $G_{0} W_{0} @$ $\mathrm{PBE}$ and evGW $W_{0} @ \mathrm{PBE}$ quasiparticle energies is displayed in Figure 7 c. $G_{0} W_{0}$ opens the too small PBE gaps but still suffers from a starting point dependence on the underlying DFT calculation. The $G_{0} W_{0}$ gaps are smaller than the ones from the partially self-consistent evGW $W_{0}$ scheme, which reduces the dependence on the DFT functional. With all three methods, the computed gaps decrease with increasing sheet size; see also Table 2. At our highest level of theory, evGW $0 \mathrm{PBE}$, the HOMO-LUMO gap changes from $3.95 \mathrm{eV}(4 \times 4)$ to $2.36 \mathrm{eV}$ $(15 \times 15)$. In other words, our calculations indicate that the gap of phosphorene nanosheets can be tuned by more than $1.5 \mathrm{eV}$ when changing the sheet area by a factor of $\sim 14$.

It is further observed that the PBE, $G_{0} W_{0}$, and evGW $W_{0}$ gaps follow a $1 / L$ behavior for the $L \times L$ sheets; see Figure $7 \mathrm{c}$. The same $1 / L$ scaling has been reported for DFT-PBE computed gaps of $1 \mathrm{D}$-periodic zigzag phosphorene ribbons, whereas $1 / L^{2}$ has been found for the gaps of their armchair analogue. ${ }^{126}$ Our phosphorene sheets feature zigzag as well as armchair edges, and we observe here, in agreement with ref 126 , the dominant scaling of the zigzag edges.

In Figure 7c, we extrapolate the gaps toward the $2 \mathrm{D}$ bulk limit of phosphorene $(L \rightarrow \infty)$. The extrapolated gaps are $0.68 \mathrm{eV}$ (PBE), $1.56 \mathrm{eV}\left(G_{0} W_{0}\right)$, and $1.76 \mathrm{eV}\left(\operatorname{evG} W_{0}\right)$. We are confident that our computed gaps of the finite phosphorene
Table 2. Fundamental Gap of Phosphorene in Electronvolts Calculated from DFT-PBE Eigenvalues and $G_{0} W_{0} @ P B E$ and evGW 0 @PBE Quasiparticle Energies ${ }^{a}$

\begin{tabular}{|c|c|c|c|c|c|}
\hline \multirow[b]{2}{*}{ method } & \multicolumn{3}{|c|}{ this work: $L \times L$ sheet } & \multicolumn{2}{|r|}{ periodic calculation } \\
\hline & $L=4$ & $L=15$ & $L=\infty$ & $\begin{array}{c}\text { this } \\
\text { work }\end{array}$ & literature \\
\hline DFT-PBE & 1.55 & 0.91 & 0.68 & 0.80 & $0.8^{112,113} 0.90^{114}$ \\
\hline$G_{0} W_{0} @ P B E$ & 3.65 & 2.13 & 1.56 & - & $\begin{array}{l}1.60,{ }^{115} 1.83,{ }^{116}{ }^{16} 2.0{ }^{113} \\
2.03,{ }^{114} 2.06^{117}\end{array}$ \\
\hline $\begin{array}{l}\text { evGW } W_{0} @ \mathrm{PBE} \\
\text { experiment }\end{array}$ & 3.95 & 2.36 & 1.76 & - & $\begin{array}{l}1.94,{ }^{118} 2.29^{114} \\
2.0,{ }^{118} 2.2^{119}\end{array}$ \\
\hline
\end{tabular}

${ }^{a}$ In this work we employ a cluster approach using $\mathrm{H}$-terminated phosphorene sheets consisting of $L \times L$ phosphorene unit cells. The extrapolated results $(L=\infty)$ obtained from Figure $7 \mathrm{c}$ are compared to calculations using periodic phosphorene cells.

sheets are of high numerical quality: In Table II (SI), we show that our gaps are well converged with respect to basis set size. Additionally, we use a highly accurate full-frequency method for the self-energy evaluation, as we have demonstrated in Section 8. However, the comparison of our extrapolated gaps to gaps from periodic $G W$ calculations or the experimentally measured gap of 2D periodic phosphorene (see Table 2) must be taken with a grain of salt. It has been reported in the literature that finite phosphorene sheets host edge states ${ }^{18,127}$ that are energetically close to the band edges. These edge states are absent in 2D 
periodic phosphorene, and hence, extrapolating the gap of finite phosphorene sheets may result in a gap that differs from the $2 \mathrm{D}$ periodic phosphorene gap. As a first sanity check, we compare the periodic DFT-PBE gap $(0.80 \mathrm{eV})$ and the DFT-PBE gap from extrapolation $(0.68 \mathrm{eV})$ (see Table 2$)$, finding a significant difference of $0.12 \mathrm{eV}$. We hypothesize that this difference also translates to $G W$ such that our gap extrapolation might underestimate the actual $G W 2 \mathrm{D}$ bulk limit by at least $0.1 \mathrm{eV}$.

While our cluster approach might suffer from a conceptual problem for the periodic limit (edge states), periodic $G W$ calculations of $2 \mathrm{D}$ systems face several computational challenges as described in ref 128 and summarized in the following. Indicative for these numerical challenges is the relatively large spread of the reported periodic $G W$ gaps of 1.6-2.1 eV $\left(G_{0} W_{0}\right)$ and 1.9-2.3 eV $\left(\mathrm{evG} W_{0}\right)$; see Table 2. These variations are most likely due to insufficiencies in the numerical treatment and lack of convergence, which has been systematically studied by Qiu et al. ${ }^{129}$ for a similar system (monolayer of $\mathrm{MoS}_{2}$ ). For the latter, the reported $G W$ gaps varied within a similar range as for phosphorene.

One of the computational challenges in 2D-periodic $G W$ calculations is the different screening parallel and perpendicular to the surface, which requires an anisotropic treatment of the singularities of $W$ at the $\Gamma$ point. ${ }^{128}$ A related aspect is that the $k$ point convergence is much slower than for three-dimensional systems, which has been also explicitly shown for phosphorene. ${ }^{114}$ An additional complication is the interaction between the $2 \mathrm{D}$ slabs in a $3 \mathrm{D}$ periodic approach with plane waves. The vacuum spacing between the repeated slabs cannot be converged out due to the long-range nature of the image charge interaction between the slabs. The correct behavior can be restored by using Coulomb truncation schemes ${ }^{129,130}$ or postprocessing corrections. ${ }^{128}$ All these issues are avoided in our cluster approach, where periodic boundary conditions are not employed.

\section{COMPUTATIONAL EFFICIENCY}

Finally, we use the phosphorene nanosheets to demonstrate the scaling and the parallel efficiency of our algorithm. As illustrated in Figure $7 \mathrm{~d}$, the $O\left(N^{2}\right)$ scaling is preserved from our previous work. ${ }^{19}$ The largest calculations were performed for the phosphorene sheets with 990 atoms $(15 \times 15$ sheet $)$, which corresponds to 6795 electrons per spin that are expanded in 25110 basis functions. The crossover between the traditional $O\left(N^{4}\right)$ implementation and the low-scaling $G W$ calculation is at around 300 atoms $[\approx 2100$ electrons per spin, $\approx 7600$ basis functions]. As shown in Figure $7 d$, the crossover point is found by extrapolation due to the high memory demands of the conventional algorithm, which practically restricts the conventional GW calculations to phosphorene sheets of 200-250 atoms. Our low-scaling approach improves also the scaling with respect to memory consumption. The conventional implementation scales $O\left(N^{3}\right)$ in memory, which is reduced to $O\left(N^{2}\right)$ in this work.

In our previous implementation of low-scaling $G W$ with the overlap metric, we reported a crossover point at 150 atoms for quasi-1D graphene nanoribbons. ${ }^{19}$ The shift to 300 atoms is because of the larger amount of three-center integrals that need to be included in the computation of $\chi_{0}(i \tau)$ in eq 22 . This is caused by three circumstances. First, sparsity conditions are only met for larger system sizes due to the $2 \mathrm{D}$ nature of the phosphorene sheets, whereas graphene nanoribbons are quasi1D systems. Second, we use in this work a Gaussian basis set
(aug-cc-pVDZ) with much smaller exponents than in ref 19. The aug-cc-pVDZ basis comprises very diffuse functions (lowest Gaussian exponent in aug-cc-pVDZ for $\mathrm{H}$ is $0.02974 \mathrm{au}$ and for $\mathrm{P}$ is $0.0343 \mathrm{au})$. For diffuse functions, fewer three-center integrals are zero than for more compact basis sets. Third, the truncated Coulomb metric is "less local" than the overlap metric used in ref 19. All three points increase the computational prefactor, which is the reason why the largest phosphorene sheet contains "only" 990 atoms, while in ref 19, we reported $G W$ calculations of a graphene nanoribbon with around 1700 atoms.

The parallel performance of our low-scaling algorithm is assessed for the $10 \times 10$ phosphorene sheet (460 atoms) employing the cc-pVDZ basis set. ${ }^{106}$ Strong scaling measurements for this system are reported in Figure 7e, where the speedup of the calculation with respect to 8 computing nodes is shown. The $G W$ calculation for the $10 \times 10$ sheet scales well up to 128 nodes (6144 processes) with a parallel efficiency of $74 \%$. Note that the $G W$ calculation for the $10 \times 10$ sheet runs also on $2-7$ nodes thanks to an iterative memory reduction scheme. ${ }^{27}$ This scheme overcomes memory bottlenecks for small node numbers by additional communication, without increasing the number of operations. Nevertheless, the additional MPI communication slightly increases the computational cost, which results in a better than ideal speed-up for larger node numbers, which do not require memory reduction. For a fair assessment of the parallel performance, we choose thus 8 nodes as the reference in Figure 7e and the cc-pVDZ basis set instead of the aug-cc-pVDZ. The latter is more diffuse and requires more memory than cc-pVDZ, triggering the memory reduction scheme also for node numbers larger than 8 .

The parallel performance and computational efficiency is also excellent for the larger phosphorene sheets. The evGW calculation for the $(15 \times 15)$ sheet $(990$ atoms $)$ was performed on 768 nodes ( $\approx 37000 \mathrm{CPU}$ cores) with a run time of $15 \mathrm{~h}$.

\section{CONCLUSION}

We have presented an accurate low-scaling $G W$ algorithm for computing quasiparticle energies in the $G W$ approximation for systems up to 1000 atoms. The algorithm achieves high accuracy by using the RI approach with the truncated Coulomb metric in combination with carefully (pre)optimized minimax grids up to 34 time and frequency points each. We have implemented the method in the open-source quantum chemistry package $\mathrm{CP}_{2} \mathrm{~K}^{90}$ and benchmarked the accuracy for HOMOs and LUMOs using the GW100 test set. The MADs with respect to the reference values from canonical $G W$ implementations are 7 and $6 \mathrm{meV}$, respectively. The benchmark studies have been extended to semicore states and unbound unoccupied states using a 24-atom phosphorene cluster. We have shown that all $G W$ quasiparticle levels in the range between $\mathrm{HOMO}-20 \mathrm{eV}$ and LUMO+20 eV agree with the highly accurate contour-deformation results from FHI-aims within $0.10 \mathrm{eV}$. The reported high accuracy together with the good scalability to 1000 atoms is yet another stepping stone toward predictive $G W$ calculations on nanostructured materials. We have demonstrated this on the example of phosphorene, showing that finite size effects can be used to engineer its band gap.

\section{ASSOCIATED CONTENT}

\section{S1 Supporting Information}

The Supporting Information is available free of charge at https://pubs.acs.org/doi/10.1021/acs.jctc.0c01282. 
Demonstration that the RI factorization in a plane-wave RI basis set is independent of the RI metric; results for the GW 100 benchmark set with 30 minimax points; input file of $\mathrm{CP} 2 \mathrm{~K}$ for the $G W 100$ test, the xyz geometry of the phosphorene cluster with 24 atoms, the customized RI basis set for hydrogen and phosphorus, and a CP2K input for a large-scale $G W$ calculation; and detailed comparison between FHI-aims and CP2K on the phosphorene sheets from Section 9 (PDF)

\section{AUTHOR INFORMATION}

\section{Corresponding Author}

Jan Wilhelm - Institute of Theoretical Physics, University of Regensburg, D-93053 Regensburg, Germany; 이이.org/ 0000-0001-8678-8246; Email: jan.wilhelm@physik.uniregensburg.de

\section{Authors \\ Patrick Seewald - Department of Chemistry, University of Zurich, CH-8057 Zurich, Switzerland \\ Dorothea Golze - Department of Applied Physics, Aalto University, FI-00076 Aalto, Finland; ○ orcid.org/0000- 0002-2196-9350}

Complete contact information is available at: https://pubs.acs.org/10.1021/acs.jctc.0c01282

\section{Funding}

The Gauss Centre for Supercomputing is acknowledged for providing computational resources on SuperMUC-NG at the Leibniz Supercomputing Centre under the Project IDs pn69mi and pn72pa. We also thank the CSC - IT Center for Science for providing computational resources. J.W. acknowledges funding from DFG SFB 1277 (Project A03). P.S. acknowledges funding by the NCCR MARVEL, funded by the Swiss National Science Foundation. D.G. acknowledges financial support by the Academy of Finland (Grant No. 316168).

\section{Notes}

The authors declare no competing financial interest.

\section{ACKNOWLEDGMENTS}

We kindly thank Mauro Del Ben, Ferdinand Evers, Jaroslav Fabian, Tobias Frank, Jürg Hutter, and Jonas Schramm for helpful discussions.

\section{REFERENCES}

(1) Hedin, L. New Method for Calculating the One-Particle Green's Function with Application to the Electron-Gas Problem. Phys. Rev. 1965, 139, A796-A823.

(2) Golze, D.; Dvorak, M.; Rinke, P. The GW Compendium: A Practical Guide to Theoretical Photoemission Spectroscopy. Front. Chem. 2019, 7, 377.

(3) Reining, L. The $G W$ approximation: content, successes and limitations. WIREs Comput. Mol. Sci. 2017, 8, No. e1344.

(4) Salpeter, E. E.; Bethe, H. A. A Relativistic Equation for BoundState Problems. Phys. Rev. 1951, 84, 1232-1242.

(5) Onida, G.; Reining, L.; Rubio, A. Electronic excitations: densityfunctional versus many-body Green's-function approaches. Rev. Mod. Phys. 2002, 74, 601.

(6) Blase, X.; Duchemin, I.; Jacquemin, D. The Bethe-Salpeter equation in chemistry: relations with TD-DFT, applications and challenges. Chem. Soc. Rev. 2018, 47, 1022-1043.

(7) Blase, X.; Duchemin, I.; Jacquemin, D.; Loos, P.-F. The BetheSalpeter Equation Formalism: From Physics to Chemistry. J. Phys. Chem. Lett. 2020, 11, 7371.
(8) Berger, J. A.; Loos, P.-F.; Romaniello, P. Potential Energy Surfaces without Unphysical Discontinuities: The Coulomb Hole Plus Screened Exchange Approach. J. Chem. Theory Comput. 2021, 17, 191-200.

(9) Çaylak, O.; Baumeier, B. Excited-State Geometry Optimization of Small Molecules with Many-Body Green's Functions Theory. J. Chem. Theory Comput. 2021, 17, 879.

(10) Aoki, T.; Ohno, K. Accurate quasiparticle calculation of x-ray photoelectron spectra of solids. J. Phys.: Condens. Matter 2018, 30, $21 \mathrm{LT} 01$.

(11) Golze, D.; Wilhelm, J.; van Setten, M. J.; Rinke, P. Core-Level Binding Energies from GW: An Efficient Full-Frequency Approach within a Localized Basis. J. Chem. Theory Comput. 2018, 14, 48564869.

(12) Golze, D.; Keller, L.; Rinke, P. Accurate Absolute and Relative Core-Level Binding Energies from GW. J. Phys. Chem. Lett. 2020, 11, 1840-1847.

(13) Keller, L.; Blum, V.; Rinke, P.; Golze, D. Relativistic correction scheme for core-level binding energies from GW. J. Chem. Phys. 2020, $153,114110$.

(14) Zhu, T.; Chan, G. K.-L. All-Electron Gaussian-Based $G_{0} W_{0}$ for Valence and Core Excitation Energies of Periodic Systems. J. Chem. Theory Comput. 2021, 17, 727.

(15) van Setten, M. J.; Caruso, F.; Sharifzadeh, S.; Ren, X.; Scheffler, M.; Liu, F.; Lischner, J.; Lin, L.; Deslippe, J. R.; Louie, S. G.; Yang, C.; Weigend, F.; Neaton, J. B.; Evers, F.; Rinke, P. GW100: Benchmarking $G_{0} W_{0}$ for Molecular Systems. J. Chem. Theory Comput. 2015, 11, 56655687.

(16) Maggio, E.; Liu, P.; van Setten, M. J.; Kresse, G. GW100: A Plane Wave Perspective for Small Molecules. J. Chem. Theory Comput. 2017, 13, 635-648.

(17) Gao, W.; Chelikowsky, J. R. Real-Space Based Benchmark of $G_{0} W_{0}$ Calculations on GW100: Effects of Semicore Orbitals and Orbital Reordering. J. Chem. Theory Comput. 2019, 15, 5299-5307.

(18) Govoni, M.; Galli, G. GW100: Comparison of Methods and Accuracy of Results Obtained with the WEST Code. J. Chem. Theory Comput. 2018, 14, 1895-1909.

(19) Wilhelm, J.; Golze, D.; Talirz, L.; Hutter, J.; Pignedoli, C. A. Toward GW Calculations on Thousands of Atoms. J. Phys. Chem. Lett. 2018, 9, 306-312.

(20) Rangel, T.; et al. Reproducibility in $G_{0} W_{0}$ calculations for solids. Comput. Phys. Commun. 2020, 255, 107242.

(21) Förster, A.; Visscher, L. Low-Order Scaling $G_{0} W_{0}$ by Pair Atomic Density Fitting. J. Chem. Theory Comput. 2020, 16, 7381-7399.

(22) Gao, W.; Chelikowsky, J. R. Accelerating Time-Dependent Density Functional Theory and GW Calculations for Molecules and Nanoclusters with Symmetry Adapted Interpolative Separable Density Fitting. J. Chem. Theory Comput. 2020, 16, 2216-2223.

(23) Del Ben, M.; da Jornada, F. H.; Canning, A.; Wichmann, N.; Raman, K.; Sasanka, R.; Yang, C.; Louie, S. G.; Deslippe, J. Large-scale GW calculations on pre-exascale HPC systems. Comput. Phys. Commun. 2019, 235, 187-195.

(24) Neuhauser, D.; Gao, Y.; Arntsen, C.; Karshenas, C.; Rabani, E.; Baer, R. Breaking the Theoretical Scaling Limit for Predicting Quasiparticle Energies: The Stochastic GW Approach. Phys. Rev. Lett. 2014, 113, 076402.

(25) Wilhelm, J.; Del Ben, M.; Hutter, J. GW in the Gaussian and Plane Waves Scheme with Application to Linear Acenes. J. Chem. Theory Comput. 2016, 12, 3623-3635.

(26) Stuke, A.; Kunkel, C.; Golze, D.; Todorović, M.; Margraf, J. T.; Reuter, K.; Rinke, P.; Oberhofer, H. Atomic structures and orbital energies of 61,489 crystal-forming organic molecules. Sci. Data 2020, 7, 58.

(27) Wilhelm, J.; Seewald, P.; Del Ben, M.; Hutter, J. Large-Scale Cubic-Scaling Random Phase Approximation Correlation Energy Calculations Using a Gaussian Basis. J. Chem. Theory Comput. 2016, $12,5851-5859$.

(28) Kim, M.; Mandal, S.; Mikida, E.; Chandrasekar, K.; Bohm, E.; Jain, N.; Li, Q.; Kanakagiri, R.; Martyna, G. J.; Kale, L.; Ismail-Beigi, S. 
Scalable GW software for quasiparticle properties using OpenAtom. Comput. Phys. Commun. 2019, 244, 427-441.

(29) Sangalli, D.; et al. Many-body perturbation theory calculations using the yambo code. J. Phys.: Condens. Matter 2019, 31, 325902.

(30) Del Ben, M.; Yang, C.; Li, Z.; da Jornada, F. H.; Louie, S.; Deslippe, J. Accelerating Large-Scale Excited-State GW Calculations on Leadership HPC Systems. 2020 SC20: International Conference for High Performance Computing, Networking, Storage, and Analysis; Los Alamitos, CA, U.S.A.; IEEE: 2020; pp 36-46.

(31) Duchemin, I.; Jacquemin, D.; Blase, X. Combining the $G W$ formalism with the polarizable continuum model: A state-specific nonequilibrium approach. J. Chem. Phys. 2016, 144, 164106.

(32) Li, J.; D’Avino, G.; Duchemin, I.; Beljonne, D.; Blase, X. Combining the Many-Body GW Formalism with Classical Polarizable Models: Insights on the Electronic Structure of Molecular Solids. J. Phys. Chem. Lett. 2016, 7, 2814-2820.

(33) Li, J.; D'Avino, G.; Duchemin, I.; Beljonne, D.; Blase, X. Accurate description of charged excitations in molecular solids from embedded many-body perturbation theory. Phys. Rev. B: Condens. Matter Mater. Phys. 2018, 97, 035108.

(34) Giustino, F.; Cohen, M. L.; Louie, S. G. GW method with the self-consistent Sternheimer equation. Phys. Rev. B: Condens. Matter Mater. Phys. 2010, 81, 115105.

(35) Umari, P.; Stenuit, G.; Baroni, S. GW quasiparticle spectra from occupied states only. Phys. Rev. B: Condens. Matter Mater. Phys. 2010, 81,115104 .

(36) Lambert, H.; Giustino, F. Ab initio Sternheimer-GW method for quasiparticle calculations using plane waves. Phys. Rev. B: Condens. Matter Mater. Phys. 2013, 88, 075117.

(37) Pham, T. A.; Nguyen, H.-V.; Rocca, D.; Galli, G. GW calculations using the spectral decomposition of the dielectric matrix: verification, validation, and comparison of methods. Phys. Rev. B: Condens. Matter Mater. Phys. 2013, 87, 155148.

(38) Govoni, M.; Galli, G. Large Scale GW calculations. J. Chem. Theory Comput. 2015, 11, 2680-2696.

(39) Schlipf, M.; Lambert, H.; Zibouche, N.; Giustino, F. SternheimerGW: A program for calculating $G W$ quasiparticle band structures and spectral functions without unoccupied states. Comput. Phys. Commun. 2020, 247, 106856.

(40) Wilson, H. F.; Gygi, F.; Galli, G. Efficient iterative method for calculations of dielectric matrices. Phys. Rev. B: Condens. Matter Mater. Phys. 2008, 78, 113303.

(41) Wilson, H. F.; Lu, D.; Gygi, F.; Galli, G. Iterative calculations of dielectric eigenvalue spectra. Phys. Rev. B: Condens. Matter Mater. Phys. 2009, 79, 245106.

(42) Friedrich, C. Tetrahedron integration method for strongly varying functions: Application to the GT self-energy. Phys. Rev. B: Condens. Matter Mater. Phys. 2019, 100, 075142.

(43) Duchemin, I.; Blase, X. Robust Analytic-Continuation Approach to Many-Body GW Calculations. J. Chem. Theory Comput. 2020, 16, $1742-1756$.

(44) Bintrim, S. J.; Berkelbach, T. C. Full-Frequency GW without Frequency. J. Chem. Phys. 2021, 154, 041101.

(45) Blase, X.; Attaccalite, C.; Olevano, V. First-principles GW calculations for fullerenes, porphyrins, phtalocyanine, and other molecules of interest for organic photovoltaic applications. Phys. Rev. B: Condens. Matter Mater. Phys. 2011, 83, 115103.

(46) Ren, X.; Rinke, P.; Blum, V.; Wieferink, J.; Tkatchenko, A.; Sanfilippo, A.; Reuter, K.; Scheffler, M. Resolution-of-identity approach to Hartree-Fock, hybrid density functionals, RPA, MP2 and GW with numeric atom-centered orbital basis functions. New J. Phys. 2012, 14, 053020 .

(47) van Setten, M. J.; Weigend, F.; Evers, F. The GW-Method for Quantum Chemistry Applications: Theory and Implementation. J. Chem. Theory Comput. 2013, 9, 232-246.

(48) Bruneval, F.; Rangel, T.; Hamed, S. M.; Shao, M.; Yang, C.; Neaton, J. B. molgw 1: Many-body perturbation theory software for atoms, molecules, and clusters. Comput. Phys. Commun. 2016, 208, 149-161.
(49) Sun, Q; et al. Recent developments in the PySCF program package. J. Chem. Phys. 2020, 153, 024109.

(50) Wilhelm, J.; Hutter, J. Periodic $G W$ calculations in the Gaussian and plane-waves scheme. Phys. Rev. B: Condens. Matter Mater. Phys. 2017, 95, 235123.

(51) Ren, X.; Merz, F.; Jiang, H.; Yao, Y.; Rampp, M.; Lederer, H.; Blum, V.; Scheffler, M. All-electron periodic $G_{0} W_{0}$ implementation with numerical atomic orbital basis functions: Algorithm and benchmarks. Phys. Rev. Materials 2021, 5, 013807.

(52) Wilhelm, J.; VandeVondele, J.; Rybkin, V. V. Dynamics of the Bulk Hydrated Electron from Many-Body Wave-Function Theory. Angew. Chem., Int. Ed. 2019, 58, 3890-3893.

(53) Iskakov, S.; Yeh, C.-N.; Gull, E.; Zgid, D. Ab initio self-energy embedding for the photoemission spectra of $\mathrm{NiO}$ and $\mathrm{MnO}$. Phys. Rev. B: Condens. Matter Mater. Phys. 2020, 102, 085105.

(54) Vlček, V.; Rabani, E.; Neuhauser, D.; Baer, R. Stochastic GW Calculations for Molecules. J. Chem. Theory Comput. 2017, 13, 49975003.

(55) Foerster, D.; Koval, P.; Sánchez-Portal, D. An $O\left(N^{3}\right)$ implementation of Hedin's $G W$ approximation for molecules. J. Chem. Phys. 2011, 135, 074105.

(56) Liu, P.; Kaltak, M.; Klimeš, J.; Kresse, G. Cubic scaling GW: Towards fast quasiparticle calculations. Phys. Rev. B: Condens. Matter Mater. Phys. 2016, 94, 165109.

(57) Duchemin, I.; Blase, X. Separable resolution-of-the-identity with all-electron Gaussian bases: Application to cubic-scaling RPA. J. Chem. Phys. 2019, 150, 174120.

(58) Kim, M.; Martyna, G. J.; Ismail-Beigi, S. Complex-time shredded propagator method for large-scale $G W$ calculations. Phys. Rev. B: Condens. Matter Mater. Phys. 2020, 101, 035139.

(59) Rojas, H. N.; Godby, R. W.; Needs, R. J. Space-Time Method for $A b$ Initio Calculations of Self-Energies and Dielectric Response Functions of Solids. Phys. Rev. Lett. 1995, 74, 1827-1830.

(60) Rieger, M. M.; Steinbeck, L.; White, I.; Rojas, H.; Godby, R. The $G W$ space-time method for the self-energy of large systems. Comput. Phys. Commun. 1999, 117, 211-228.

(61) Vahtras, O.; Almlöf, J.; Feyereisen, M. Integral approximations for LCAO-SCF calculations. Chem. Phys. Lett. 1993, 213, 514.

(62) Duchemin, I.; Li, J.; Blase, X. Hybrid and Constrained Resolution-of-Identity Techniques for Coulomb Integrals. J. Chem. Theory Comput. 2017, 13, 1199-1208.

(63) Eshuis, H.; Yarkony, J.; Furche, F. Fast computation of molecular random phase approximation correlation energies using resolution of the identity and imaginary frequency integration. J. Chem. Phys. 2010, $132,234114$.

(64) Golze, D.; Benedikter, N.; Iannuzzi, M.; Wilhelm, J.; Hutter, J. Fast evaluation of solid harmonic Gaussian integrals for local resolution-of-the-identity methods and range-separated hybrid functionals. J. Chem. Phys. 2017, 146, 034105.

(65) Obara, S.; Saika, A. Efficient recursive computation of molecular integrals over Cartesian Gaussian functions. J. Chem. Phys. 1986, 84, 3963-3974.

(66) Ahlrichs, R. A simple algebraic derivation of the Obara-Saika scheme for general two-electron interaction potentials. Phys. Chem. Chem. Phys. 2006, 8, 3072-3077.

(67) Guidon, M. High performance Hartree-Fock exchange for large and condensed phase systems. Ph.D. thesis, University of Zuerich, 2010; pp 86-89; DOI: 10.5167/uzh-44716.

(68) Luenser, A.; Schurkus, H. F.; Ochsenfeld, C. VanishingOverhead Linear-Scaling Random Phase Approximation by Cholesky Decomposition and an Attenuated Coulomb-Metric. J. Chem. Theory Comput. 2017, 13, 1647-1655.

(69) Del Ben, M.; Hutter, J.; VandeVondele, J. Electron Correlation in the Condensed Phase from a Resolution of Identity Approach Based on the Gaussian and Plane Waves Scheme. J. Chem. Theory Comput. 2013, 9, 2654-2671.

(70) Rybkin, V. V.; VandeVondele, J. Spin-Unrestricted SecondOrder Møller-Plesset (MP2) Forces for the Condensed Phase: From 
Molecular Radicals to F-Centers in Solids. J. Chem. Theory Comput. 2016, 12, 2214-2223.

(71) Weigend, F.; Häser, M.; Patzelt, H.; Ahlrichs, R. RI-MP2: optimized auxiliary basis sets and demonstration of efficiency. Chem. Phys. Lett. 1998, 294, 143-152.

(72) Gui, X.; Holzer, C.; Klopper, W. Accuracy Assessment of GW Starting Points for Calculating Molecular Excitation Energies Using the Bethe-Salpeter Formalism. J. Chem. Theory Comput. 2018, 14, 21272136.

(73) Holzer, C.; Klopper, W. Ionized, electron-attached, and excited states of molecular systems with spin-orbit coupling: Two-component GW and Bethe-Salpeter implementations. J. Chem. Phys. 2019, 150, 204116.

(74) Holzer, C.; Teale, A. M.; Hampe, F.; Stopkowicz, S.; Helgaker, T.; Klopper, W. GW quasiparticle energies of atoms in strong magnetic fields. J. Chem. Phys. 2019, 150, 214112.

(75) Rybkin, V. V. Sampling Potential Energy Surfaces in the Condensed Phase with Many-Body Electronic Structure Methods. Chem. - Eur. J. 2020, 26, 362-368.

(76) Hutter, J.; Wilhelm, J.; Rybkin, V. V.; Del Ben, M.; VandeVondele, J. MP2- and RPA-Based Ab Initio Molecular Dynamics and Monte Carlo Sampling. In Handbook of Materials Modeling: Methods: Theory and Modeling; Andreoni, W., Yip, S., Eds.; Springer: 2018.

(77) Jung, Y.; Sodt, A.; Gill, P. M.; Head-Gordon, M. Auxiliary basis expansions for large-scale electronic structure calculations. Proc. Natl. Acad. Sci. U. S. A. 2005, 102, 6692-6697.

(78) Jung, Y.; Shao, Y.; Head-Gordon, M. Fast evaluation of scaled opposite spin second-order Møller-Plesset correlation energies using auxiliary basis expansions and exploiting sparsity. J. Comput. Chem. 2007, 28, 1953-1964.

(79) Reine, S.; Tellgren, E.; Krapp, A.; Kjærgaard, T.; Helgaker, T.; Jansik, B.; Høst, S.; Salek, P. Variational and robust density fitting of four-center two-electron integrals in local metrics. J. Chem. Phys. 2008, 129, 104101.

(80) Heyd, J.; Scuseria, G. E.; Ernzerhof, M. Hybrid functionals based on a screened Coulomb potential. J. Chem. Phys. 2003, 118, 82078215.

(81) Dutoi, A. D.; Head-Gordon, M. A Study of the Effect of Attenuation Curvature on Molecular Correlation Energies by Introducing an Explicit Cutoff Radius into Two-Electron Integrals. J. Phys. Chem. A 2008, 112, 2110-2119.

(82) Graf, D.; Beuerle, M.; Schurkus, H. F.; Luenser, A.; Savasci, G.; Ochsenfeld, C. Accurate and Efficient Parallel Implementation of an Effective Linear-Scaling Direct Random Phase Approximation Method. J. Chem. Theory Comput. 2018, 14, 2505-2515.

(83) Beuerle, M.; Graf, D.; Schurkus, H. F.; Ochsenfeld, C. Efficient calculation of beyond RPA correlation energies in the dielectric matrix formalism. J. Chem. Phys. 2018, 148, 204104.

(84) Beuerle, M.; Ochsenfeld, C. Low-scaling analytical gradients for the direct random phase approximation using an atomic orbital formalism. J. Chem. Phys. 2018, 149, 244111.

(85) Graf, D.; Beuerle, M.; Ochsenfeld, C. Low-Scaling SelfConsistent Minimization of a Density Matrix Based Random Phase Approximation Method in the Atomic Orbital Space. J. Chem. Theory Comput. 2019, 15, 4468-4477.

(86) Merlot, P.; Kjærgaard, T.; Helgaker, T.; Lindh, R.; Aquilante, F.; Reine, S.; Pedersen, T. B. Attractive electron-electron interactions within robust local fitting approximations. J. Comput. Chem. 2013, 34, 1486-1496.

(87) Ihrig, A. C.; Wieferink, J.; Zhang, I. Y.; Ropo, M.; Ren, X.; Rinke, P.; Scheffler, M.; Blum, V. Accurate localized resolution of identity approach for linear-scaling hybrid density functionals and for manybody perturbation theory. New J. Phys. 2015, 17, 093020.

(88) Golze, D.; Iannuzzi, M.; Hutter, J. Local Fitting of the KohnSham Density in a Gaussian and Plane Waves Scheme for Large-Scale Density Functional Theory Simulations. J. Chem. Theory Comput. 2017, $13,2202-2214$.
(89) Pritchard, B. P.; Altarawy, D.; Didier, B.; Gibson, T. D.; Windus, T. L. New Basis Set Exchange: An Open, Up-to-Date Resource for the Molecular Sciences Community. J. Chem. Inf. Model. 2019, 59, 48144820.

(90) Kühne, T. D.; et al. CP2K: An electronic structure and molecular dynamics software package - Quickstep: Efficient and accurate electronic structure calculations. J. Chem. Phys. 2020, 152, 194103.

(91) CP2K github repository. https://github.com/cp2k/cp2k (accessed December, 2020).

(92) Borštnik, U.; VandeVondele, J.; Weber, V.; Hutter, J. Sparse matrix multiplication: The distributed block-compressed sparse row library. Parallel Comput 2014, 40, 47-58.

(93) Kaltak, M.; Klimeš, J.; Kresse, G. Low Scaling Algorithms for the Random Phase Approximation: Imaginary Time and Laplace Transforms. J. Chem. Theory Comput. 2014, 10, 2498-2507.

(94) Braess, D.; Hackbusch, W. Approximation of $1 / \mathrm{x}$ by exponential sums in $[1, \infty)$. SIAM J. Numer. Anal. 2005, 25, 685-697.

(95) Kaltak, M.; Kresse, G. Minimax isometry method: A compressive sensing approach for Matsubara summation in many-body perturbation theory. Phys. Rev. B: Condens. Matter Mater. Phys. 2020, 101, 205145.

(96) Blum, V.; Gehrke, R.; Hanke, F.; Havu, P.; Havu, V.; Ren, X.; Reuter, K.; Scheffler, M. Ab initio molecular simulations with numeric atom-centered orbitals. Comput. Phys. Commun. 2009, 180, 21752196.

(97) Golze, D. Dataset in NOMAD repository "Low-scaling GW: GW100 and phosphorene; 2020; DOI: 10.17172/NOMAD/ 2021.01.15-1.

(98) Lippert, G.; Hutter, J.; Parrinello, M. The Gaussian and augmented-plane-wave density functional method for ab initio molecular dynamics simulations. Theor. Chem. Acc. 1999, 103, 124140.

(99) Perdew, J. P.; Burke, K.; Ernzerhof, M. Generalized Gradient Approximation Made Simple. Phys. Rev. Lett. 1996, 77, 3865-3868.

(100) Vidberg, H. J.; Serene, J. W. Solving the Eliashberg equations by means of N-point Padé approximants. J. Low Temp. Phys. 1977, 29, 179-192.

(101) Weigend, F.; Furche, F.; Ahlrichs, R. Gaussian basis sets of quadruple zeta valence quality for atoms $\mathrm{H}-\mathrm{Kr}$. J. Chem. Phys. 2003, $119,12753-12762$.

(102) Hättig, C. Optimization of auxiliary basis sets for RI-MP2 and RI-CC2 calculations: Core-valence and quintuple-zeta basis sets for $\mathrm{H}$ to Ar and QZVPP basis sets for Li to Kr. Phys. Chem. Chem. Phys. 2005, $7,59-66$

(103) Grimme, S.; Antony, J.; Ehrlich, S.; Krieg, H. A consistent and accurate $\mathrm{ab}$ initio parametrization of density functional dispersion correction (DFT-D) for the 94 elements H-Pu. J. Chem. Phys. 2010, 132, 154104.

(104) Goedecker, S.; Teter, M.; Hutter, J. Separable dual-space Gaussian pseudopotentials. Phys. Rev. B: Condens. Matter Mater. Phys. 1996, 54, 1703-1710.

(105) VandeVondele, J.; Hutter, J. Gaussian basis sets for accurate calculations on molecular systems in gas and condensed phases. $J$. Chem. Phys. 2007, 127, 114105.

(106) Dunning, T. H. Gaussian basis sets for use in correlated molecular calculations. I. The atoms boron through neon and hydrogen. J. Chem. Phys. 1989, 90, 1007-1023.

(107) Woon, D. E.; Dunning, T. H. Gaussian basis sets for use in correlated molecular calculations. IV. Calculation of static electrical response properties. J. Chem. Phys. 1994, 100, 2975-2988.

(108) Kendall, R. A.; Dunning, T. H.; Harrison, R. J. Electron affinities of the first-row atoms revisited. Systematic basis sets and wave functions. J. Chem. Phys. 1992, 96, 6796-6806.

(109) Weigend, F.; Köhn, A.; Hättig, C. Efficient use of the correlation consistent basis sets in resolution of the identity MP2 calculations. J. Chem. Phys. 2002, 116, 3175-3183.

(110) Gatti, M.; Panaccione, G.; Reining, L. Effects of Low-Energy Excitations on Spectral Properties at Higher Binding Energy: The Metal-Insulator Transition of $\mathrm{VO}_{2}$. Phys. Rev. Lett. 2015, 114, 116402. 
(111) Zhou, J. S.; Kas, J. J.; Sponza, L.; Reshetnyak, I.; Guzzo, M.; Giorgetti, C.; Gatti, M.; Sottile, F.; Rehr, J. J.; Reining, L. Dynamical effects in electron spectroscopy. J. Chem. Phys. 2015, 143, 184109.

(112) Frank, T.; Derian, R.; Tokár, K.; Mitas, L.; Fabian, J.; Stich, I. Many-Body Quantum Monte Carlo Study of 2D Materials: Cohesion and Band Gap in Single-Layer Phosphorene. Phys. Rev. X 2019, 9, 011018 .

(113) Tran, V.; Soklaski, R.; Liang, Y.; Yang, L. Layer-controlled band gap and anisotropic excitons in few-layer black phosphorus. Phys. Rev. B: Condens. Matter Mater. Phys. 2014, 89, 235319.

(114) Rasmussen, F. A.; Schmidt, P. S.; Winther, K. T.; Thygesen, K. S. Efficient many-body calculations for two-dimensional materials using exact limits for the screened potential: Band gaps of $\mathrm{MoS}_{2}, h-\mathrm{BN}$, and phosphorene. Phys. Rev. B: Condens. Matter Mater. Phys. 2016, 94, 155406.

(115) Rudenko, A. N.; Katsnelson, M. I. Quasiparticle band structure and tight-binding model for single- and bilayer black phosphorus. Phys. Rev. B: Condens. Matter Mater. Phys. 2014, 89, 201408.

(116) Jiang, Z.; Liu, Z.; Li, Y.; Duan, W. Scaling Universality between Band Gap and Exciton Binding Energy of Two-Dimensional Semiconductors. Phys. Rev. Lett. 2017, 118, 266401.

(117) Ferreira, F.; Ribeiro, R. M. Improvements in the $G W$ and BetheSalpeter-equation calculations on phosphorene. Phys. Rev. B: Condens. Matter Mater. Phys. 2017, 96, 115431.

(118) Liang, L.; Wang, J.; Lin, W.; Sumpter, B. G.; Meunier, V.; Pan, M. Electronic Bandgap and Edge Reconstruction in Phosphorene Materials. Nano Lett. 2014, 14, 6400-6406.

(119) Wang, X.; Jones, A. M.; Seyler, K. L.; Tran, V.; Jia, Y.; Zhao, H.; Wang, H.; Yang, L.; Xu, X.; Xia, F. Highly anisotropic and robust excitons in monolayer black phosphorus. Nat. Nanotechnol. 2015, 10, 517-521.

(120) Liu, H.; Neal, A. T.; Zhu, Z.; Luo, Z.; Xu, X.; Tománek, D.; Ye, P. D. Phosphorene: An Unexplored 2D Semiconductor with a High Hole Mobility. ACS Nano 2014, 8, 4033-4041.

(121) Li, L.; Yu, Y.; Ye, G. J.; Ge, Q.; Ou, X.; Wu, H.; Feng, D.; Chen, X. H.; Zhang, Y. Black phosphorus field-effect transistors. Nat. Nanotechnol. 2014, 9, 372-377.

(122) Ling, X.; Wang, H.; Huang, S.; Xia, F.; Dresselhaus, M. S. The renaissance of black phosphorus. Proc. Natl. Acad. Sci. U. S. A. 2015, 112, 4523-4530.

(123) Castellanos-Gomez, A. Black Phosphorus: Narrow Gap, Wide Applications. J. Phys. Chem. Lett. 2015, 6, 4280-4291.

(124) Vlček, V.; Rabani, E.; Baer, R.; Neuhauser, D. Nonmonotonic band gap evolution in bent phosphorene nanosheets. Phys. Rev. Materials 2019, 3, 064601.

(125) Brooks, J.; Weng, G.; Taylor, S.; Vlček, V. Stochastic many-body perturbation theory for Moire states in twisted bilayer phosphorene. J. Phys.: Condens. Matter 2020, 32, 234001.

(126) Tran, V.; Yang, L. Scaling laws for the band gap and optical response of phosphorene nanoribbons. Phys. Rev. B: Condens. Matter Mater. Phys. 2014, 89, 245407.

(127) Peng, X.; Copple, A.; Wei, Q. Edge effects on the electronic properties of phosphorene nanoribbons. J. Appl. Phys. 2014, 116, 144301.

(128) Freysoldt, C.; Eggert, P.; Rinke, P.; Schindlmayr, A.; Scheffler, M. Screening in two dimensions: $G W$ calculations for surfaces and thin films using the repeated-slab approach. Phys. Rev. B: Condens. Matter Mater. Phys. 2008, 77, 235428.

(129) Qiu, D. Y.; da Jornada, F. H.; Louie, S. G. Screening and manybody effects in two-dimensional crystals: Monolayer $\mathrm{MoS}_{2}$. Phys. Rev. B: Condens. Matter Mater. Phys. 2016, 93, 235435.

(130) Ismail-Beigi, S. Truncation of periodic image interactions for confined systems. Phys. Rev. B: Condens. Matter Mater. Phys. 2006, 73, 233103. 